\title{
A review of the process of knowledge transfer and use of evidence in reproductive and child health in Ghana
}

\author{
Gordon Abekah-Nkrumah" ${ }^{1 *}$, Sombié Issiaka², Lokossou Virgil² and Johnson Ermel ${ }^{2}$
}

\begin{abstract}
Background: The paper carries out a situational analysis to examine the production, dissemination and utilisation of reproductive and child health-related evidence to inform policy formulation in Ghana's health sector.

Methods: The study used Wald's model of knowledge production, transfer and utilisation as a conceptual model to collect relevant data via interviews and administration of questionnaire to a network of persons who either previously or currently hold policy-relevant positions in Ghana's health sector. Additional data was also gathered through a scoping review of the knowledge transfer and research utilisation literature, existing reproductive and child health policies, protocols and guidelines and information available on the websites of relevant institutions in Ghana's health sector.

Results: The findings of the study suggest that the health sector in Ghana has major strengths (strong knowledge production capacity, a positive environment for the promotion of evidence-informed policy) and opportunities (access to major donors who have the resources to fund good quality research and access to both local and international networks for collaborative research). What remains a challenge, however, is the absence of a robust institutional-wide mechanism for collating research needs and communicating these to researchers, communicating research findings in forms that are friendlier to policy-makers and the inability to incorporate funding for research into the budget of the health sector.
\end{abstract}

Conclusion: The study concludes, admonishing the Ministry of Health and its agencies to leverage on the existing strengths and opportunities to address the identified challenges.

Keywords: Knowledge transfer, Evidence, Reproductive and child health

\section{Background}

Evidence-based practice or evidence-informed policy-making generally refers to systematic efforts to ensure that research evidence becomes an important input into policy-making [1]. This has variously been referred to as knowledge translation, knowledge transfer, knowledge exchange, research utilisation, implementation, diffusion and dissemination [2]. Evidence-informed policy-making has assumed increased importance in several arenas of policy-making [3, 4]. In the area of health policy, the weight placed on evidence-informed policy is even much greater, with the reason that it leads to

\footnotetext{
*Correspondence: gabekah-nkrumah@ug.edu.gh

'Department of Public Administration and Health Services Management, University of Ghana Business School, P. O. Box 72, Legon, Accra, Ghana Full list of author information is available at the end of the article
}

optimal allocation and fair distribution of resources, responds to scientific and technological advances and consequently improves health outcomes [5]. Indeed, there are a couple of high profile documents that have emphasised the importance of evidence-based policy/knowledge transfer in the healthcare arena. For example, WHO and the Lord Darzi report on England National Health Service have all emphasised the need for closer collaboration between users and producers of evidence to ensure that practice is evidence informed [6].

The idea that virtually all forms of policy should be based on strong scientific evidence can be traced to the establishment of the evidence-based medicine (EBM) framework. At the core of EBM is the use of clinical evidence (resulting from scientific research) to guide clinical practice. The growth of EBM has transcended clinical practice and

(C) The Author(s). 2018 Open Access This article is distributed under the terms of the Creative Commons Attribution 4.0 International License (http://creativecommons.org/licenses/by/4.0/), which permits unrestricted use, distribution, and 
greatly influenced the call for non-clinicians (policy-makers, government officials and programme managers) to abandon policy development approaches that rely heavily on common sense, popular support and political ideology in favour of approaches that are primarily based on scientific facts/evidence generated through appropriate and robust scientific research. It is not uncommon for discussions about evidence-informed policy to generate debate about what constitutes evidence. Generally, evidence can be operationalised to mean facts (actual or asserted) gained through observation or experiences and used to support a conclusion [7]. The National Institute for Health and Clinical Excellence (NICE) further argues that evidence can either be scientific or colloquial [8]. According to NICE, scientific evidence arises from explicit (codified and propositional), systematic (use of transparent and unambiguous methods for codification), and replicable (use of methods that can reproduce results in similar circumstances) scientific methods. On the contrary, colloquial evidence arises from expert testimony or comments from practitioners and stakeholders that may be crucial in complementing scientific evidence. Within the innovation literature, evidence is also argued to include experiences or received wisdom of individuals [9]. It is important however to emphasise that, among the different facts used to support a policy or conclusion, the most reliable is argued to be scientific evidence [10-12]. It is therefore not surprising that stronger health systems around the world (both developed and developing) are believed to be those whose health policies are informed by high quality scientific research evidence $[1,5]$.

Notwithstanding the importance of using scientific research evidence to guide the formulation and implementation of health policies, there is evidence in the research utilisation literature to suggest the existence of a major gap between available research evidence and actual practice (i.e. health policy and clinical practice) [13]. The research evidence to utilisation gap is much worse in low- to middle-income countries, partly due to weak capacity (skill set and systems) to carry out policy-relevant research and ability to translate research findings into a form that can be easily utilised by policy-makers [14]. Besides the issues of capacity, the underdeveloped nature of health systems in low- to middle-income countries such as Ghana, coupled with the generally low levels of investments in health, may also be responsible. At the micro level, it is equally argued that the propensity of healthcare organisations to use research evidence in policy is determined by their ability to put in place formal and informational structures that drives organisational learning and norms, and value the importance of evidence in decision-making [15-19]. The micro and macro level weaknesses enumerated above may possibly explain the low utilisation of research evidence in health policy-making in many developing countries.
Although Ghana's investment in health has improved over the years and is regarded as one of the best in the sub-Saharan African region, it compares unfavourably to other developing countries. For example, health expenditure (HE) as a percentage of Gross Domestic Product (GDP) and HE per capita increased from 3\% in 2000 to 5.4\% in 2013 and USD 82.4 in 2000 to USD 214.2 in 2013, respectively. Ghana's HE as a percentage of GDP and HE per capita in 2013 compares favourably to Kenya (4.5\% and USD 44.5), Nigeria (3.5 and USD 115), The Gambia (6\% and USD 28.9), Uganda (9.8 and USD 59.1) and Mali (7.1 and USD 53.3). Although Rwanda's HE as a percentage of GDP is 2.5 percentage points higher than Ghana, their HE per capita of USD 83 is lower compared to Ghana [20].

Ghana's health sector has witnessed appreciable progress in several areas over the last one and half decades. Although Ghana did not meet the Millennium Development Goals target on maternal and child health (i.e. MDGs 4 and 5), key outcome indicators in the area of reproductive and child health $(\mathrm{RCH})$ have improved, such as a reduction in the national maternal mortality rate of 49\%, from 760/100,000 live births in 1990 to 380/ 100,000 live births in 2013 [21]. Indeed, Ghana's 2013 maternal mortality rate can be considered very low compared to that of neighbouring or other African countries such as Nigeria $(560 / 100,000)$, Niger $(630 / 100,000)$ and Sierra Leone $(1100 / 100,000)$ over the same period [21]. Consumption of reproductive health inputs has also improved tremendously. The report of the 2014 Ghana Demographic and Health Survey (GDHS) suggested that the percentage of women receiving antenatal care from a skilled provider increased from $82 \%$ in 1988 to $97 \%$ in 2014 , with the attendance of four or more antenatal visits also increasing from $78 \%$ in 2008 to $87 \%$ in 2014 [22]. In addition, $78 \%$ of women having given birth in the 5 years preceding the 2014 GDHS received protection against neonatal tetanus, with women delivering in a health facility increasing from $42 \%$ in 1988 to $73 \%$ in 2014. The GDHS 2014 report also suggested that 8 in 10 mothers received postnatal check-up within the crucial first 2 days after delivery.

Infant and child health has also improved over the years. For example, infant mortality and under-five mortality have declined by $28 \%$ and $44 \%$, respectively, for the period 1998 to 2014. Additionally, the 2014 GDHS suggested that neonatal mortality and perinatal mortality stood at 29/1000 and 38/1000 live births, respectively, and that the percentage of children with basic vaccination coverage increased from $47 \%$ in 1998 to $77 \%$ in 2014. Although the percentage of children (aged 1223 months) with low birth weight (less than $2.5 \mathrm{~kg}$ ) is $10 \%$, children under-five who are stunted, wasted or underweight dropped from $35 \%, 8 \%$ and $18 \%$, respectively, in 2003 to $19 \%, 5 \%$ and $11 \%$, respectively, in 2014 [22]. 
The substantial progress made in Ghana's health sector has often been linked to the adoption of sector-wide approaches in the 1990s, which ushered in systematic approaches to policy formulation and implementation in Ghana's health sector [23-25]. For example, the formulation and implementation of comprehensive and robust medium-term plans (i.e. the 5-Year Programme of Works (POWs)), starting from 1997 to date, are all products of the Ghana sector-wide approaches. There have been four 5-Year POWs (i.e. 19972001, 2002-2006, 2007-2011 and 2014-2017) since 1997. Most importantly, the POWs meant the formulation of programme-specific policies (for example, $\mathrm{RCH}$ ) to achieve the objectives of the POWs at the national level.

What is clear and unambiguous about the POWs and other programme-specific policies (i.e. $\mathrm{RCH}$ policies) in the health sector is the clear, transparent, participatory and robust processes used in their development. On the contrary, the extent to which these policies are informed by existing scientific research evidence is either unclear or has rarely featured in Ghana's health policy literature. The current paper therefore carries out a situational analysis to examine the process of knowledge production, transfer and use of scientific research evidence in the formulation of $\mathrm{RCH}$ policies in Ghana. For the purposes of this paper, $\mathrm{RCH}$ is defined to cover maternal, newborn and child health $(\mathrm{MNCH})$.

\section{Conceptual model}

The knowledge transfer and innovation diffusion literature abounds in several theoretical frameworks that aim to explain the process of knowledge transfer and eventual use of knowledge [14, 26-28]. For example, Roger's Diffusion of Innovations theory has been used extensively in the last 20 years to explain the process of transferring knowledge into practice, especially in clinical practice, healthcare organisations and in health policy-making [29]. In addition, several authors, through systematic reviews of the existing knowledge transfer and research utilisation literature [30-36], have developed conceptual frameworks meant to explain the process of knowledge transfer and utilisation of research evidence in decision-making. Evidence from recent systematic reviews suggest the existence of as many as 63 different theoretical models and frameworks on knowledge transfer from fields such as healthcare, social care and management $[26,37]$. There is also a section of the knowledge transfer/research utilisation literature that has focused on examining factors (knowledge characteristics, organisational characteristics, environmental characteristics, individual characteristics, etc.) that predict, facilitate or hinder the utilisation of research evidence $[6,28,38]$. Notwithstanding the abundance of theories and frameworks on knowledge transfer and research utilisation in this literature, the diverse nature and sheer depth of this literature makes it extremely difficult for practitioners and researchers alike to determine what constitutes an appropriate model [39]. Besides, several of these models are mostly unrefined and untested, thereby raising justifiable questions about their suitability for use in the design and evaluation of knowledge transfer interventions or understanding the research utilisation process [6]. A key criticism of most of the existing models is that they are very narrow and hardly cover the broader sociological processes in knowledge transfer [6].

To better understand the process of knowledge transfer and use of evidence in decision-making, a framework that captures the broader sociological explanation of knowledge transfer and use of research evidence has recently been developed based on a comprehensive systematic review of the existing knowledge transfer literature [6]. Although the framework has not been empirically tested just as a large chunk of those before it, its appeal arises due to the fact that it is recent and tends to combine the components of 28 different models that either wholly or partly explains the process of knowledge transfer [6]. The framework is made up of five components as explained below (Fig. 1).

- Problem identification and communication - This deals with channels used by users to communicate problems to researchers.

- Knowledge/research development and selection This deals with the knowledge or research to be transferred and attributes or characteristics that will enhance successful transfer of knowledge. Within the literature, key activities considered to be crucial at this stage of the knowledge transfer process include producing, synthesising and adapting to new knowledge.

- Analysis of context - This is part of the knowledge transfer process and looks at factors that may constrain or promote the transfer of knowledge.

- The knowledge transfer activity or intervention This is often the most common component of the knowledge transfer process and involves the actual activities undertaken to transfer knowledge.

- Knowledge/research utilisation - This looks at the actual use of knowledge or research findings transferred.

The order in which the components of the framework are listed does not in any way suggest that they happen in a linear fashion. The proponents of the framework argue that knowledge transfer does not happen in a linear fashion, but in a complex, dynamic and multidirectional fashion [6]. This view has support in the existing literature, in that researcher to policy-maker interactions constitute a key ingredient to successful knowledge transfer [40, 41]. However, for simplicity and application to $\mathrm{RCH}$ in Ghana, our analysis will be conducted as if the process occurs in a 


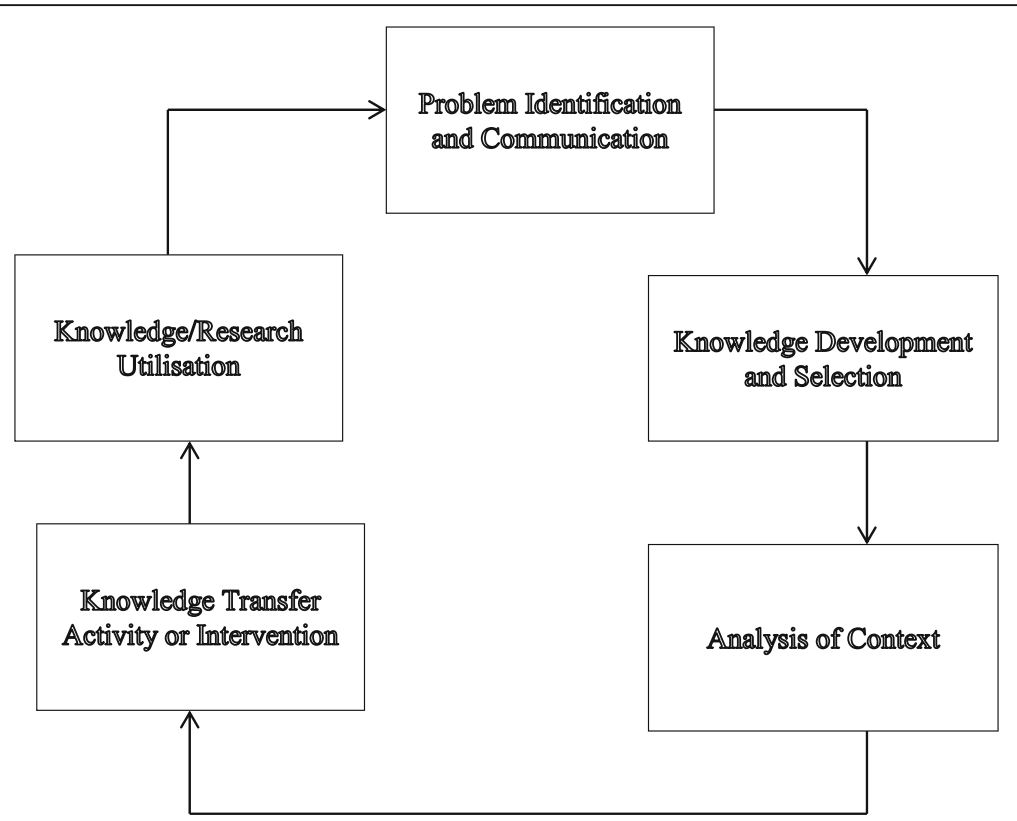

Fig. 1 Knowledge Transfer and Research Utilisation Framework (KTRUF). Source: Constructed based on Ward et al. [6]

linear form. Thus, the above five-component framework will constitute the basis for examining processes for the production, transfer and use of research evidence to inform $\mathrm{RCH}$ policy formulation in Ghana.

\section{Methods}

Using a qualitative research method, the paper conducts a situational analysis of the process of knowledge transfer and utilisation of research evidence to inform $\mathrm{RCH}$ policy in Ghana. Data for the paper was gathered through a two-stage process. The first stage focused on collecting initial data for analysis. The first step of the first stage reviewed grey literature (i.e. policies, protocols and practice guidelines; Tables 1 and 2) that could potentially provide information on the use of evidence to inform policy. This was followed with interviews and discussions with persons who either previously held or currently hold a senior level position (Director, Deputy Director, Programme Officer, etc.) in the GHS or Ministry of Health (MOH). The discussions and interviews focused on understanding the capacity of the health sector to produce, disseminate and use research evidence to inform $\mathrm{RCH}$ policy.

Additional data was also acquired through the review of information provided on the website of $\mathrm{MOH}$ and its agencies, specifically, GHS and related departments. These include the Navrongo Health Research Centre (NHRC), the Kintampo Health Research Centre (KHRC) and the Dodowa Health Research Centre (DHRC). The website review focused on gathering information on the capacity of the research centres to produce evidence and convert the evidence to a form that can easily be used by policy-makers. In addition, the websites of the GHS research centres (https://mamaye.org/welcome-e4a-mamaye), a popular website with informative content on $\mathrm{RCH}$ issues in Ghana and other West African countries was also reviewed. Additionally, RCH-related literature on Ghana was reviewed to gather evidence on the capacity of Ghana's health sector to generate scientific evidence to support policy-making in $\mathrm{RCH}$. This was done via a search through recognised public health-related databases and publishers (BMC, Elsevier, Oxford, PubMed, African Journals Online and Global Health Archives) and Google Scholar using the following search topics: (1) knowledge transfer and health policy; (2) evidence-informed health policy; (3) evidence and maternal health policy in Ghana; (4) evidence and child health policy in Ghana; (5) evidence and newborn health policy in Ghana; and (6) reproductive and child health intervention scale-up in Ghana.

Based on the above search criteria, peer-reviewed journal articles on RCH published in English since 2000, were retrieved. Overall, 77 out of a total of 534 articles retrieved were selected and reviewed. The 77 were selected on the basis of relevance and are made up of (1) 39 articles on RCH-related evidence in Ghana with at least one author being a staff member of $\mathrm{MOH} / \mathrm{GHS}$ (Table 3); (2) 28 articles on RCH-related evidence in Ghana authored by researchers outside of $\mathrm{MOH} / \mathrm{GHS}$ (Table 4); and (3) 10 articles on scaling-up of $\mathrm{RCH}$-related projects in Ghana (Table 5).

In the second stage, a draft report based on data collected in the first stage was presented to a meeting of 36 participants drawn from Ghana's health sector (i.e. mainly policy-makers/managers/senior officials within 


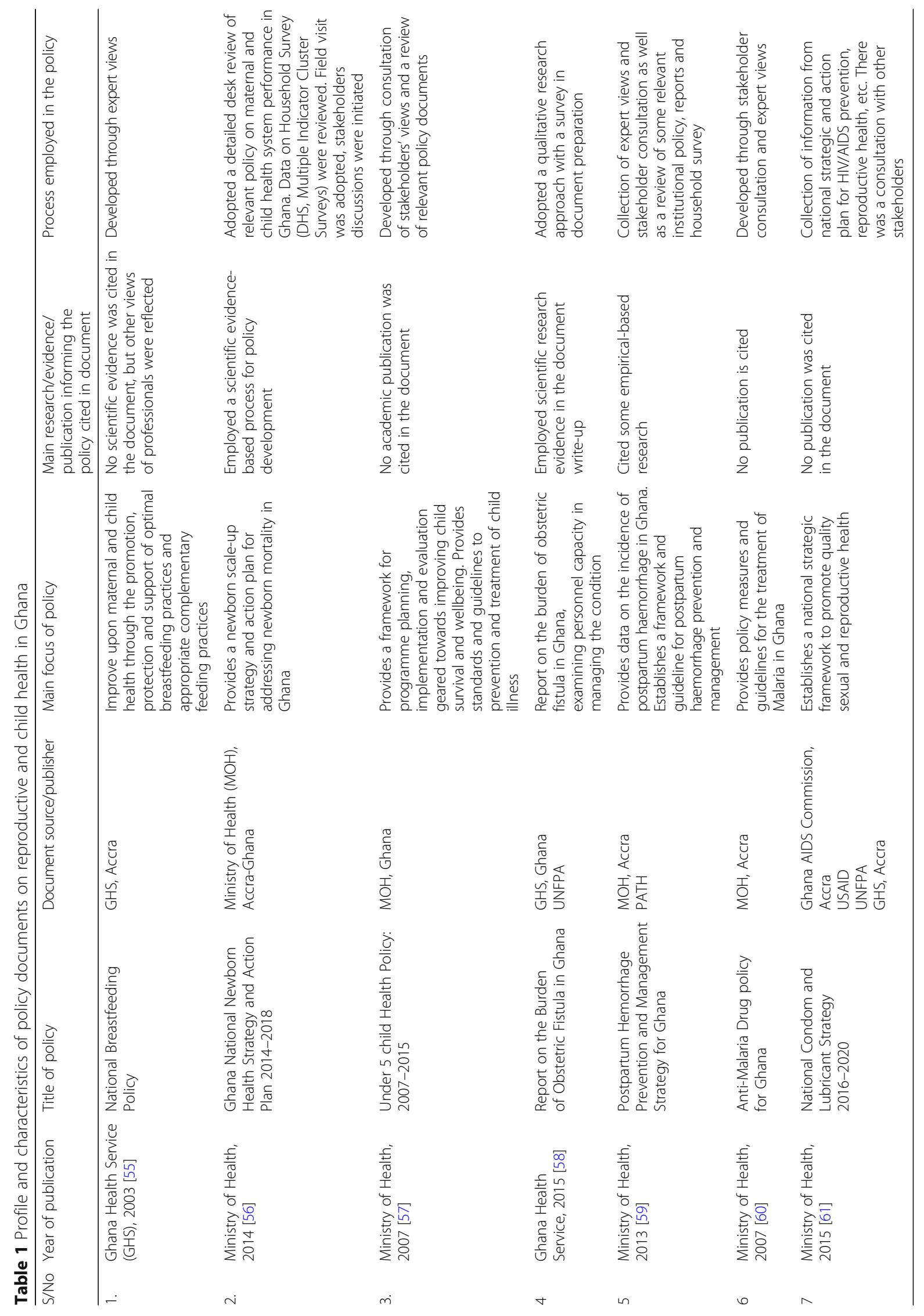




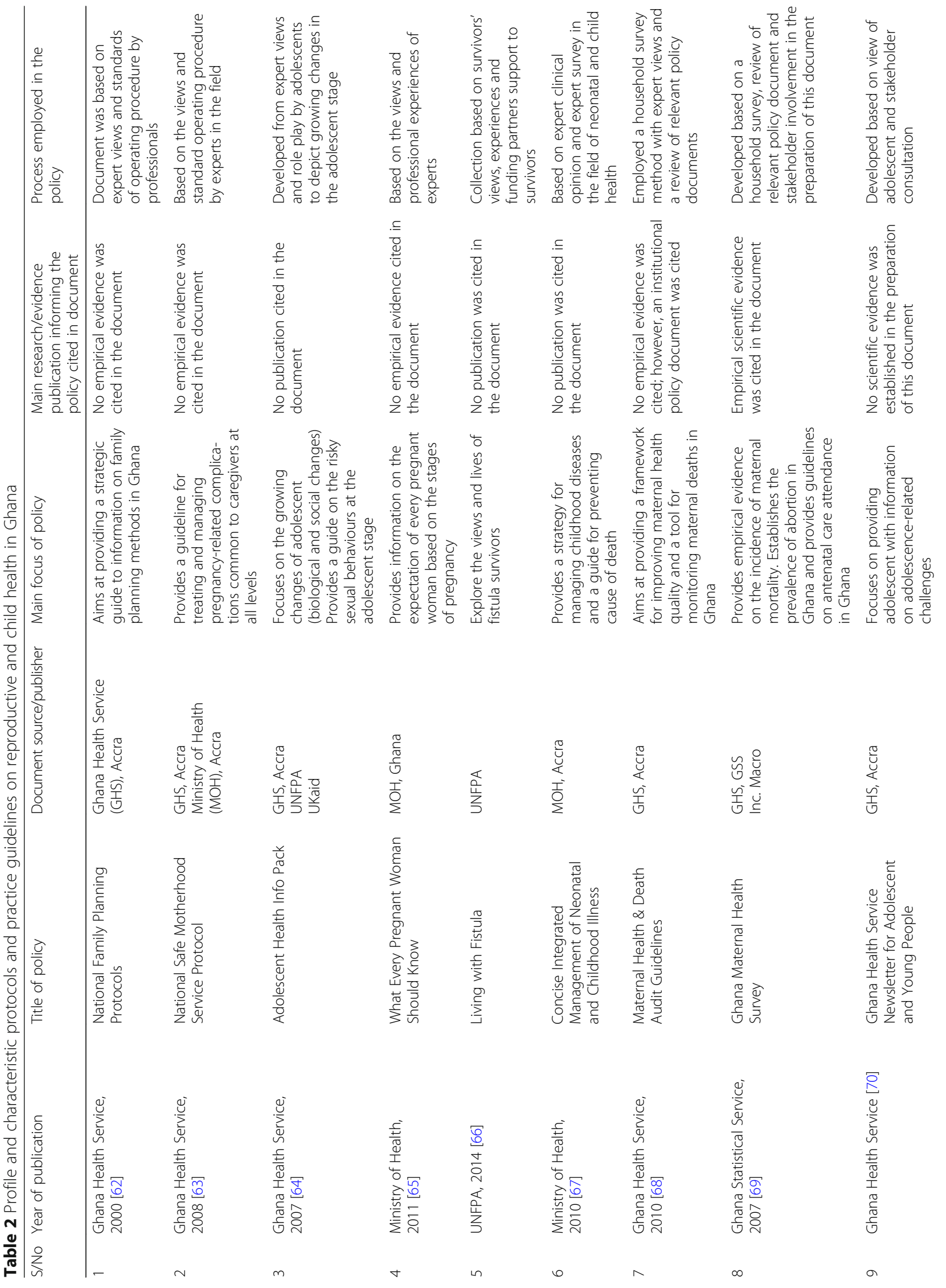




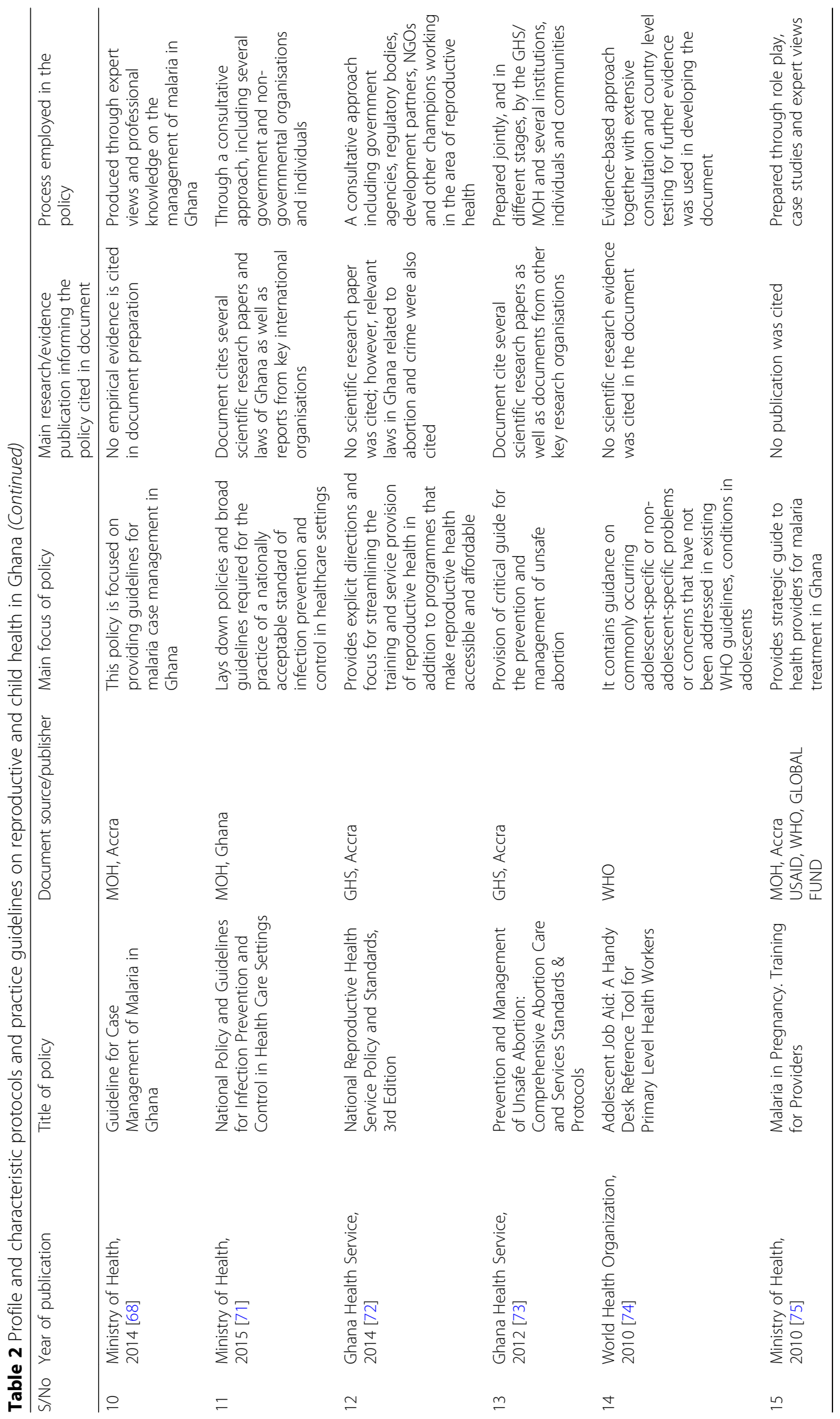




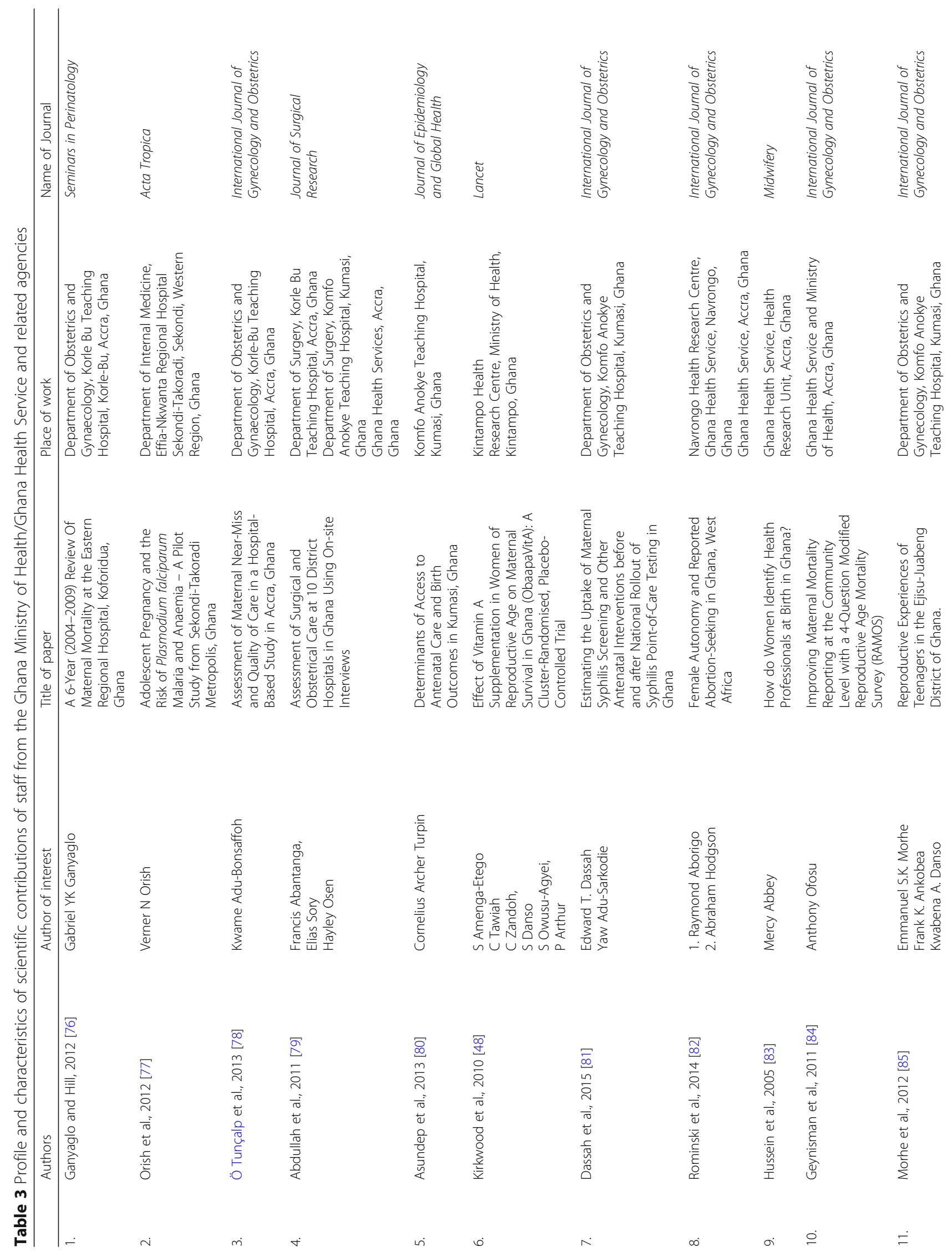




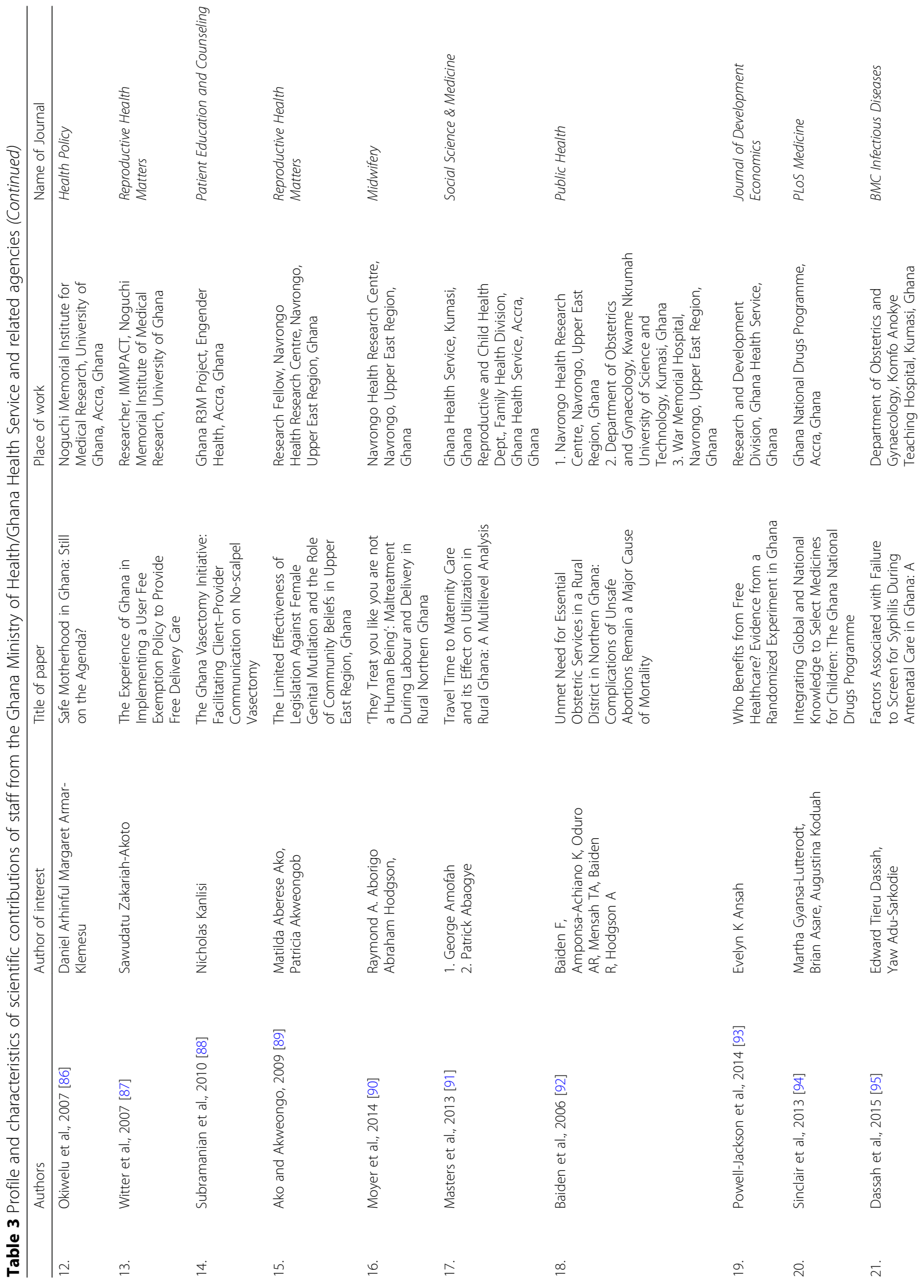




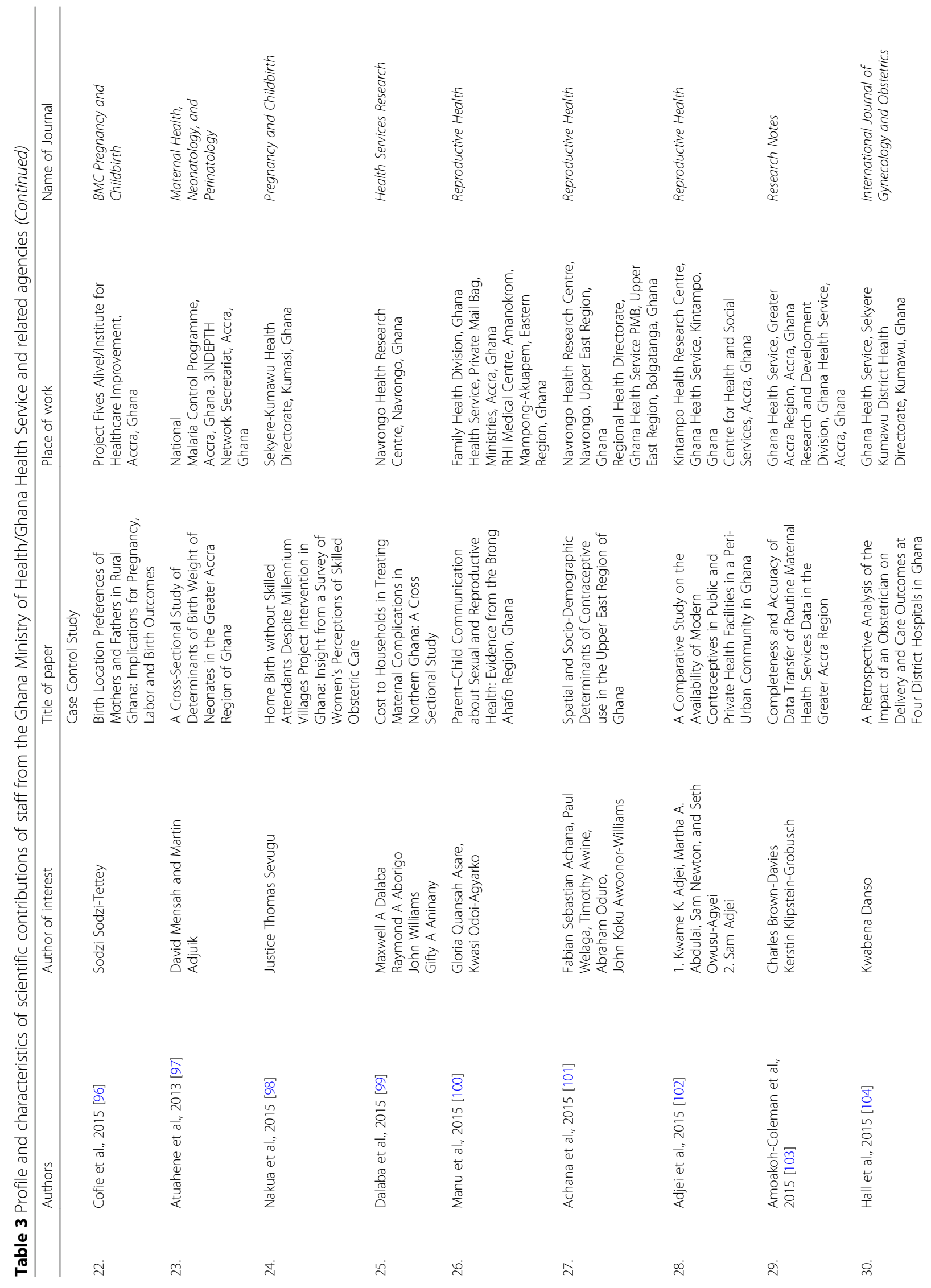




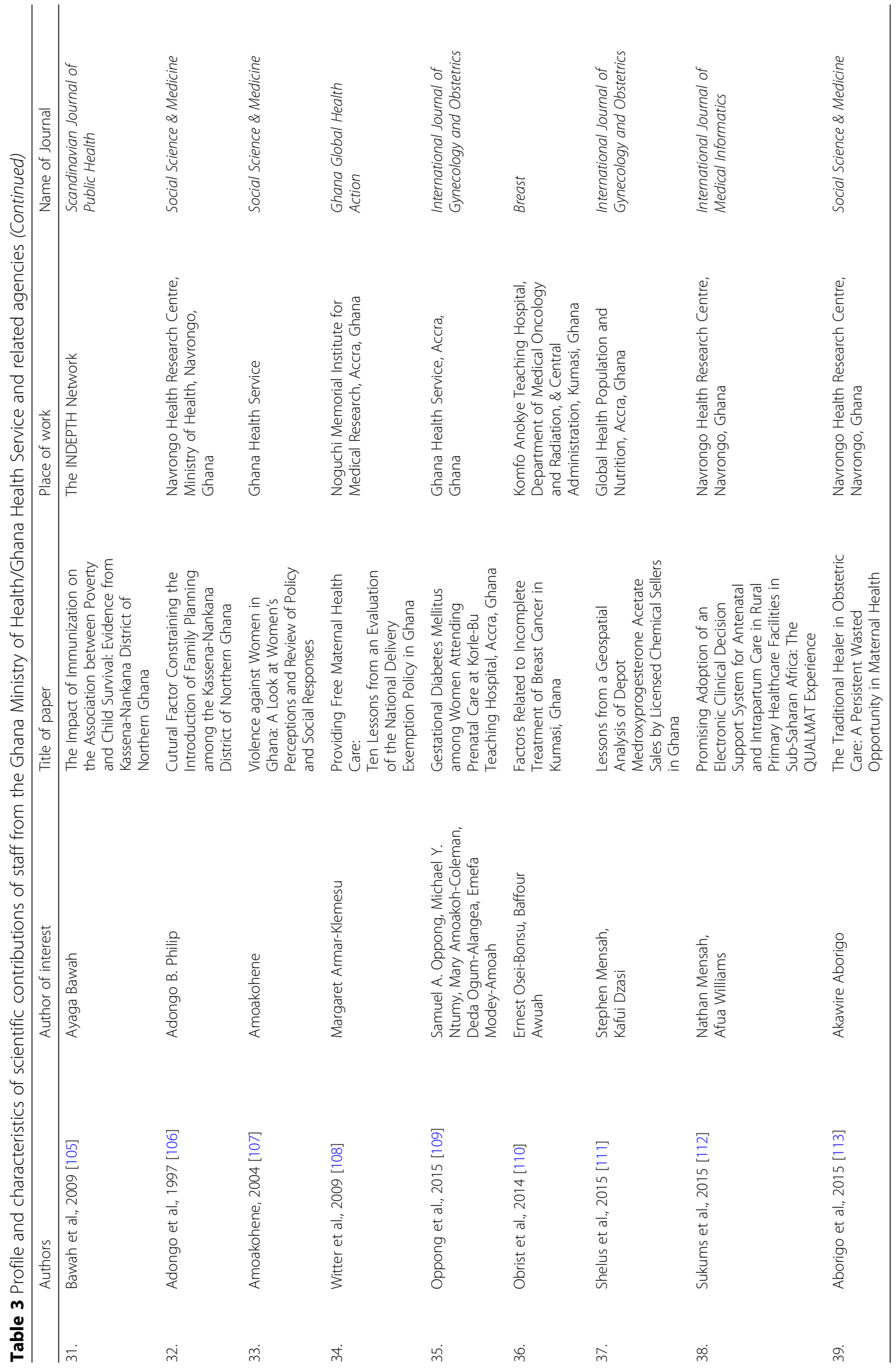




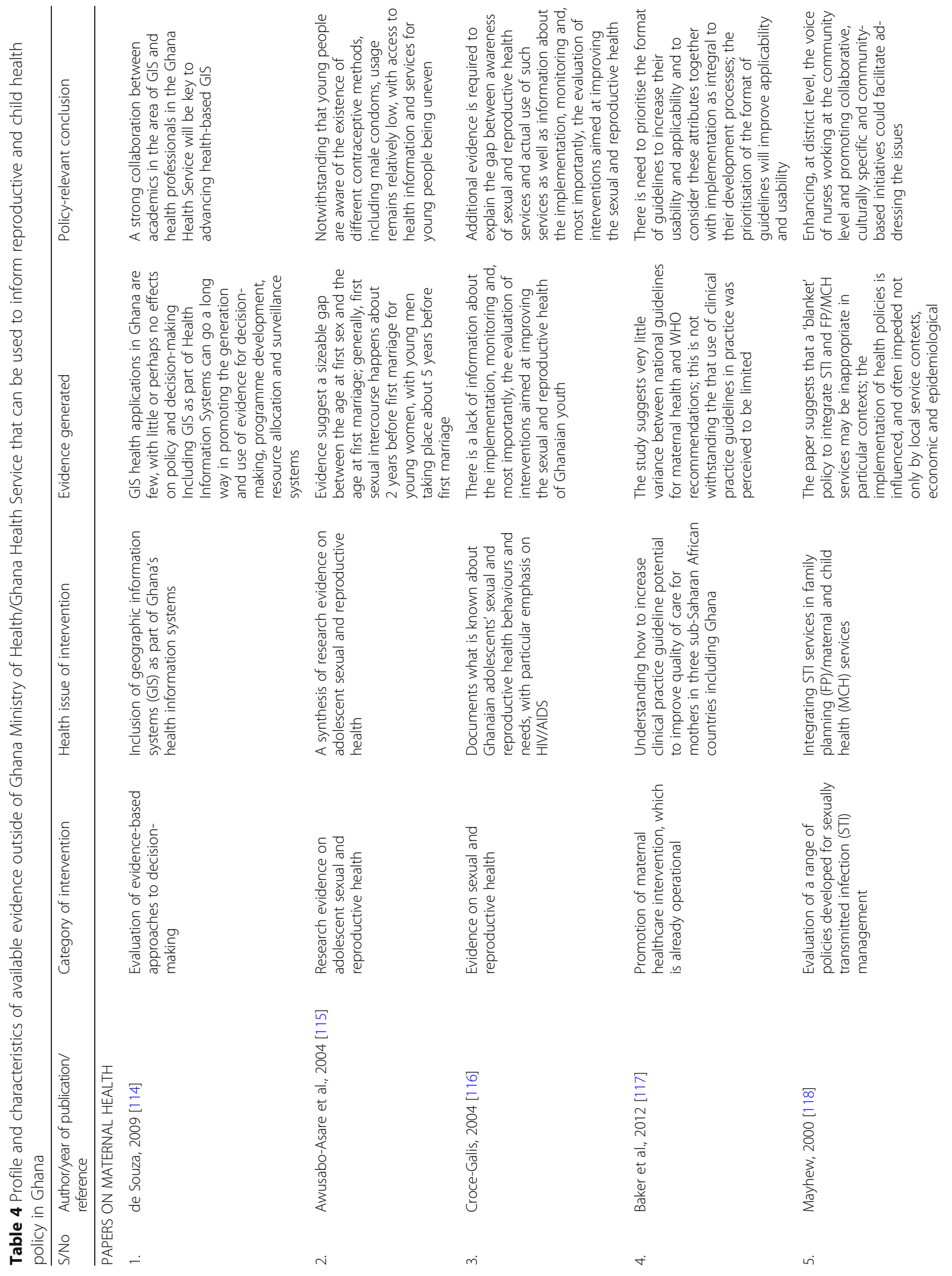




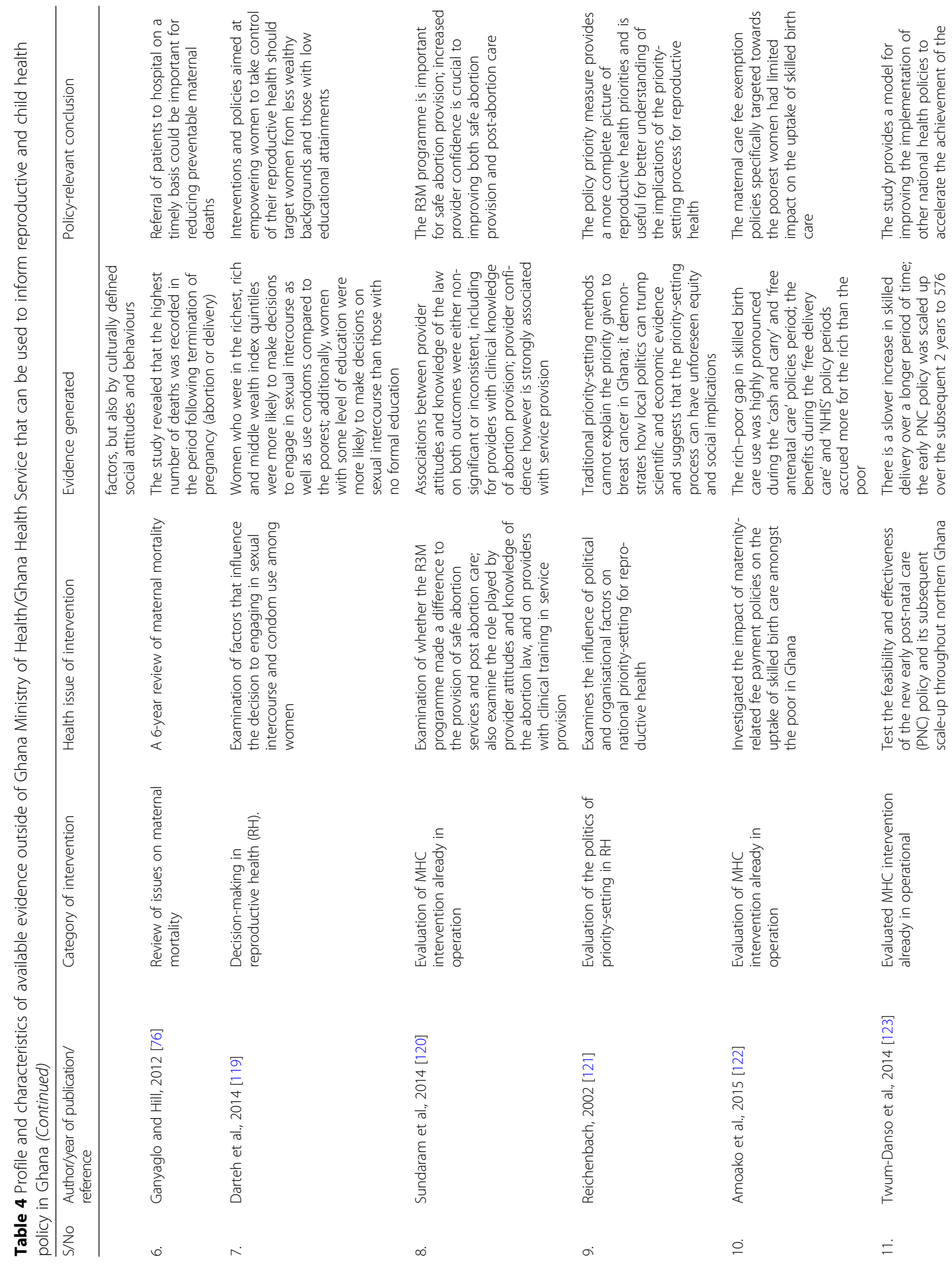




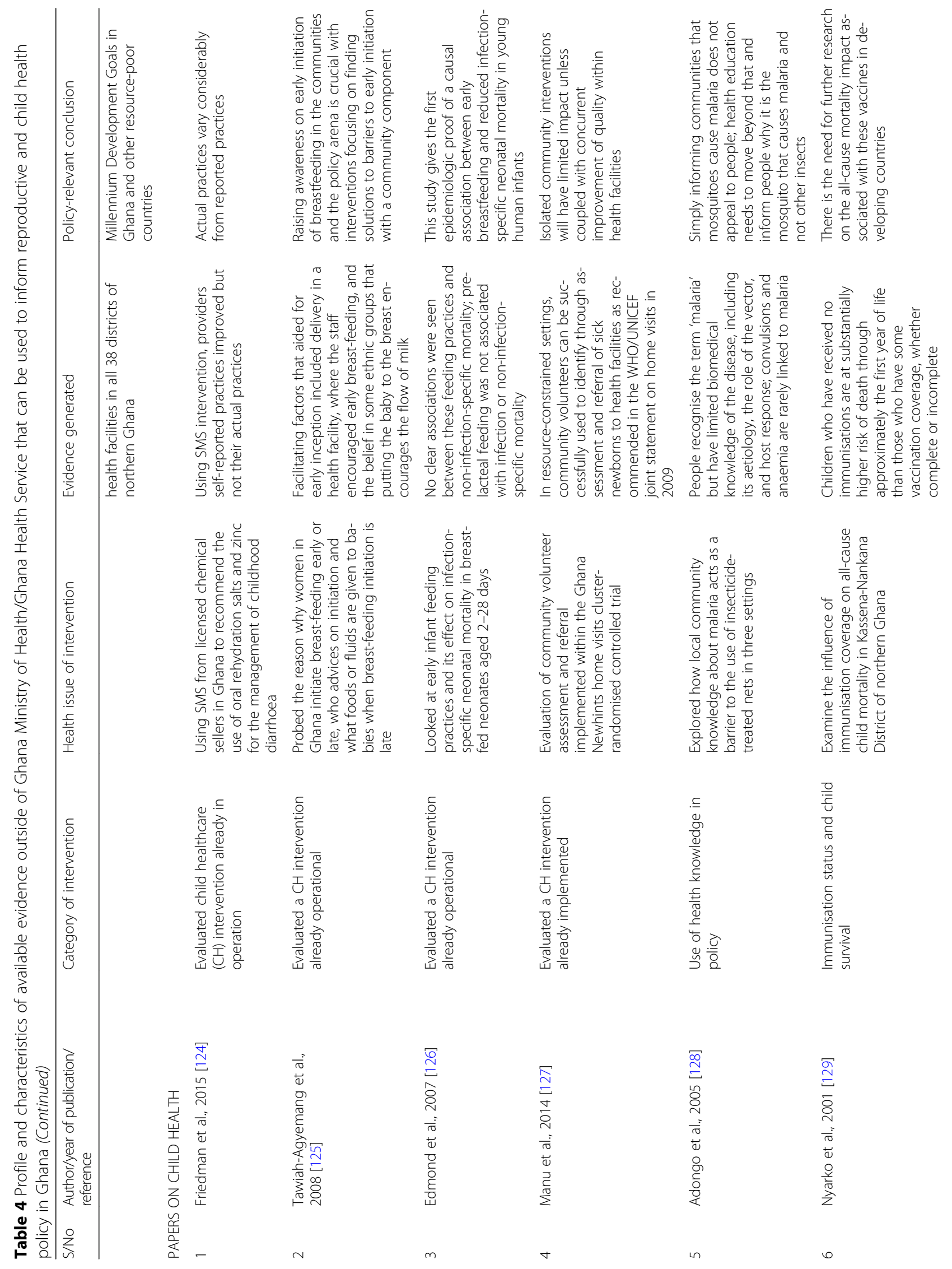




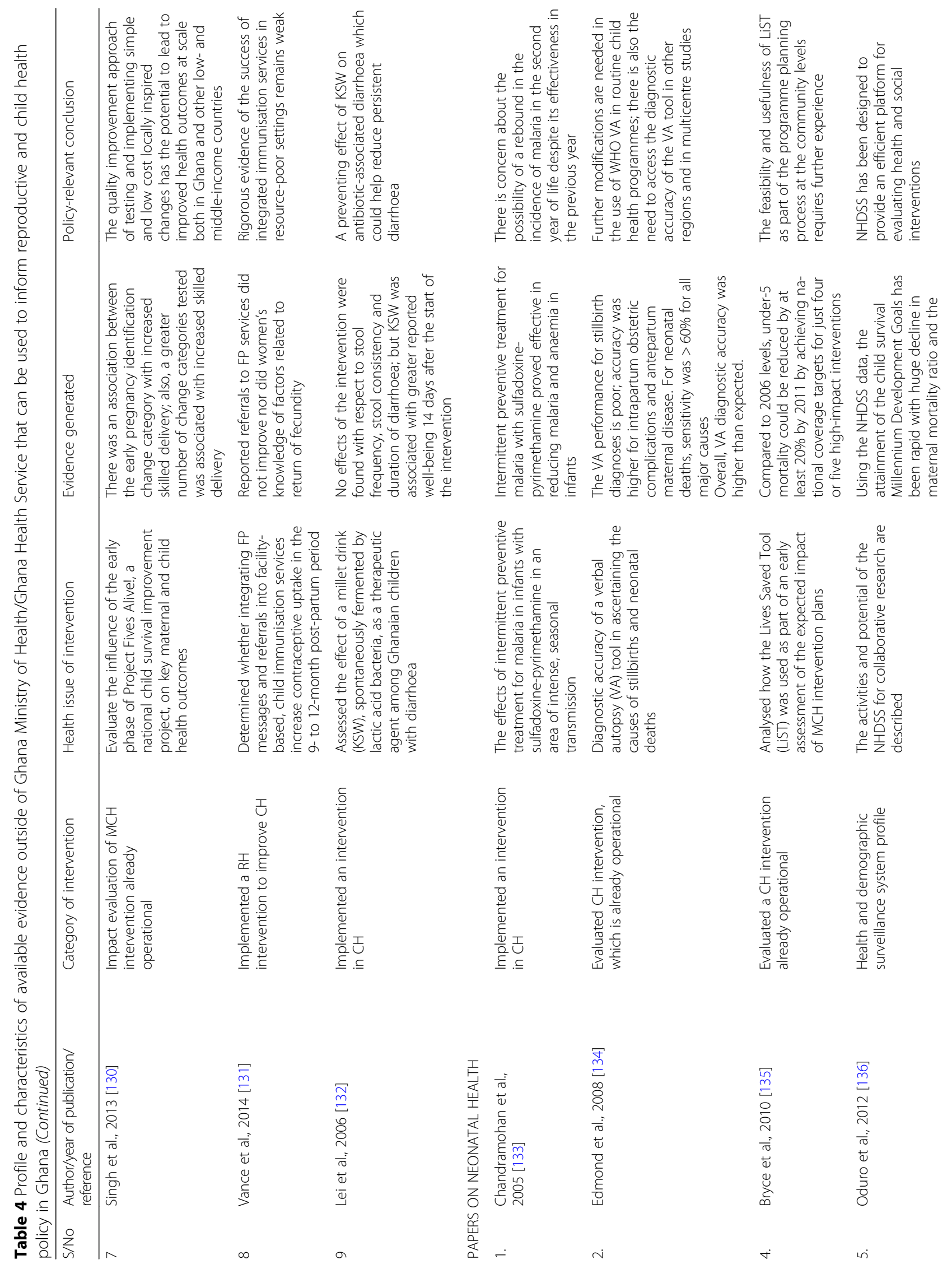




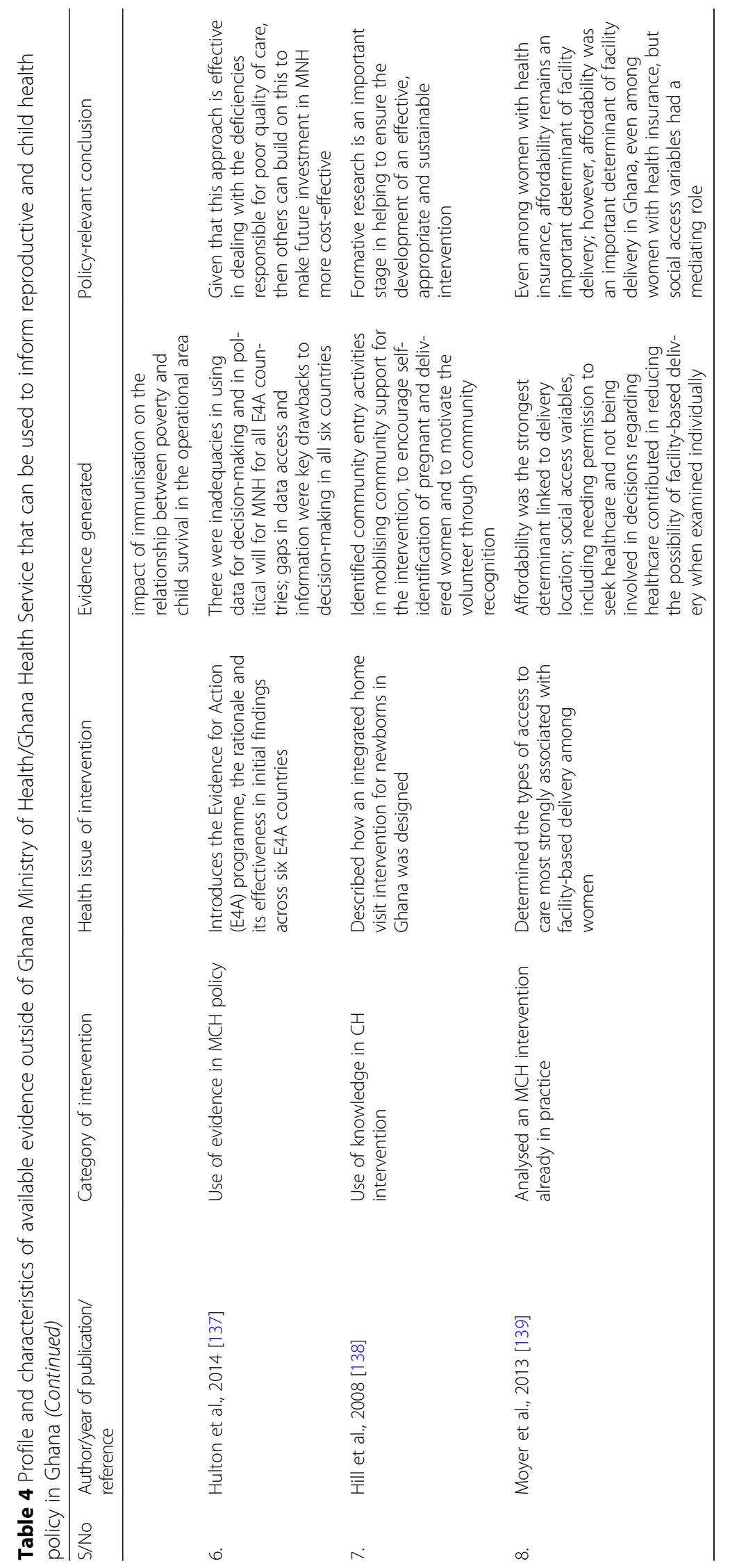




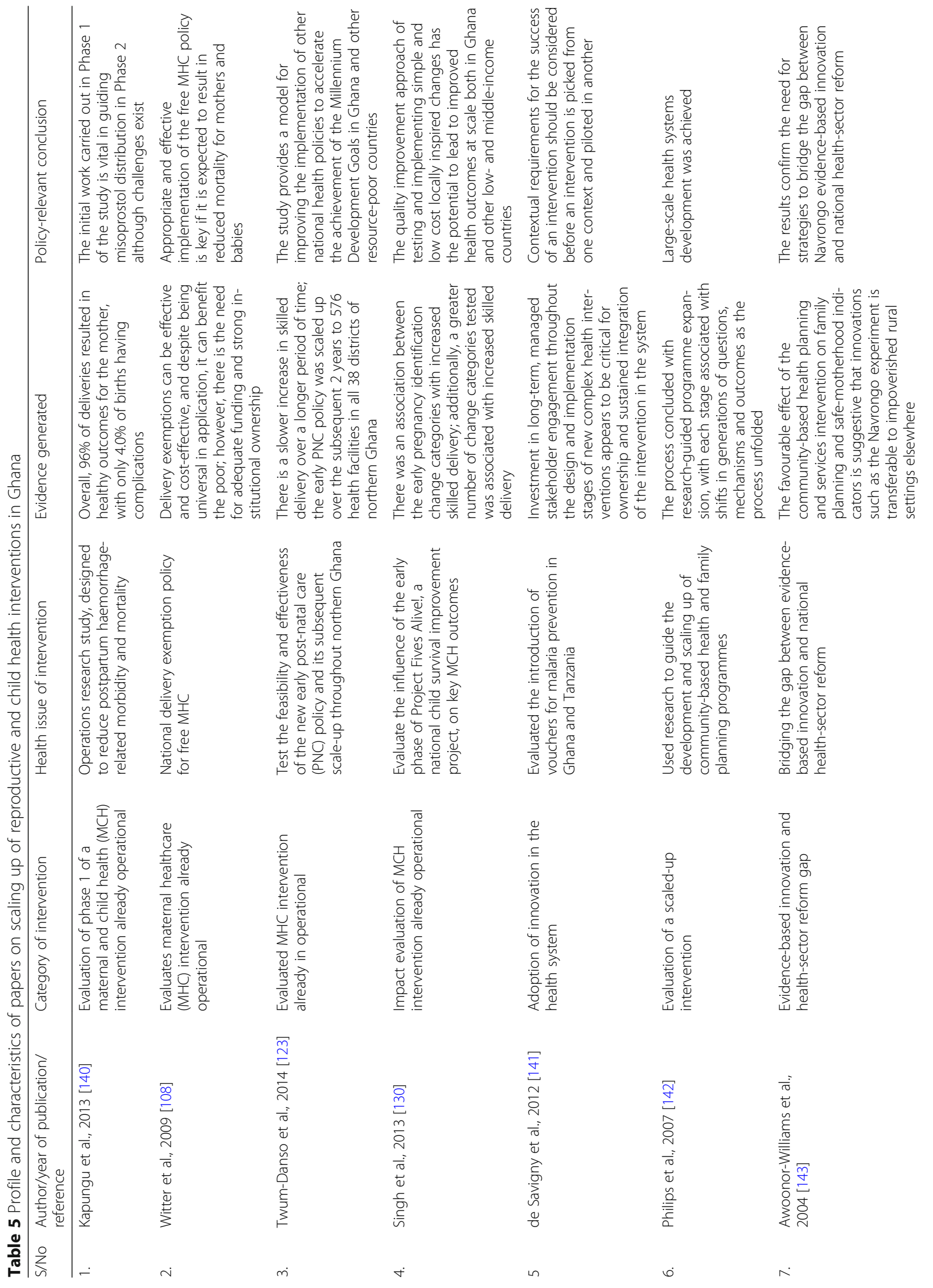




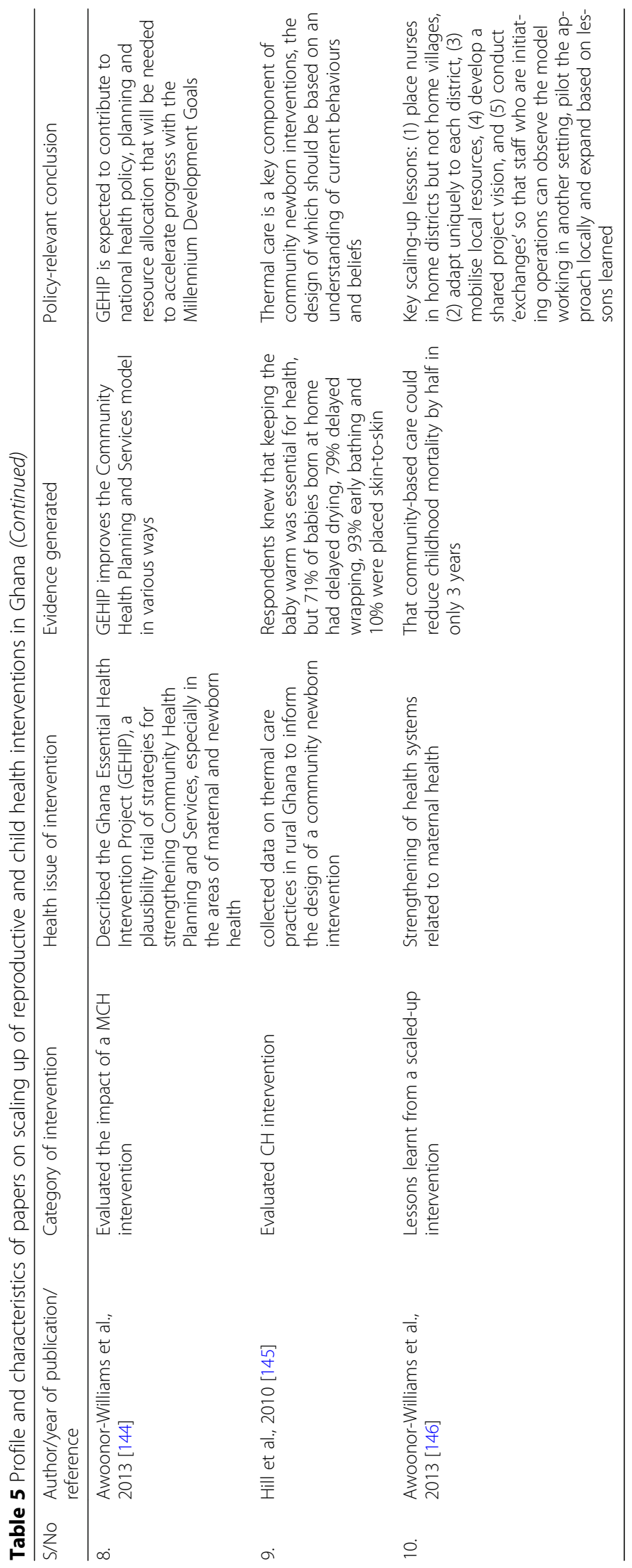


the $\mathrm{MOH}$ and related agencies, local and international NGOs in the health sector, donors and academia) and working in $\mathrm{RCH}$ for comments. The comments provided were used to amend the draft report. In addition to the comments, a questionnaire was administered to participants of the stakeholder meeting, to captured respondent data in relation to official designation attributes; knowledge and application of information communication technology; knowledge of the policy-making process; capacity to use evidence; knowledge of policy and policy-making processes related to $\mathrm{MNCH}$; acquisition of research evidence relevant to $\mathrm{MNCH}$; assessment of the validity, quality and application of research evidence relevant to $\mathrm{MNCH}$; ability to adapt formats of research results to provide information useful to decision-makers in $\mathrm{MNCH}$; and application of evidence in decision-making relevant to $\mathrm{MNCH}$.

A total of 15 participants (GHS $n=7, \mathrm{MOH} n=1$, NGOs $n=4$, Donors and Academia $n=4$ ) responded to the questionnaire. Besides the questionnaire, we solicited and incorporated the views of the 36 participants on what they believe promotes the use of evidence in their workplace through group discussions. Specifically, participants deliberated on issues related to aptitudes, skills, institutional environment, platforms/mechanisms, sources of evidence, nature of evidence, opportunity for the use of evidence and support needed to use evidence for policy formulation. Although participants in the group discussion and respondents to the questionnaire were in the stakeholder meeting as representatives of their respective organisations, their participation was based on their consent and not on compulsion. Data from the questionnaire and group discussions was analysed and reflected in the draft report. It is important to emphasise that data collected was analysed and presented along the themes/components of knowledge transfer and research utilisation framework presented in Fig. 1.

\section{Results}

In this section, we present the findings of the study structured around the components of the knowledge transfer and research utilisation framework.

\section{Problem identification and communication}

In agreement with the core issues identified under this component, we sought to elucidate, from the data collected, the procedure used to identify $\mathrm{RCH}$ research needs and how those needs were communicated to researchers. Discussions with key managers within the health sector, as well as informal networks of individuals who have once worked in policy positions in the health sector, suggest that there is currently no institutional structure either at the level of the $\mathrm{MOH}$ or GHS that identifies research needs of units and directorates and channels them to researchers either within the health sector or outside of it.

This notwithstanding, a few units collate their research needs and incorporate this information into their annual plans to be performed when funds are available either through Government of Ghana budget or from donor funds. Within the $\mathrm{RCH}$ unit of the GHS, for example, the practice has been to aggregate research needs and incorporate these as part of the programme of work for the year, with the actual research carried out when funds become available either through the Government of Ghana budget or donor-funded programmes. Besides the above, there are also mechanisms that make it possible for research needs of the sector to be communicated to donors so that funds are made available to undertake such research or incorporated into the objectives of existing research funded by donors.

The mixed picture given by respondents interviewed and participants of the group discussion seems to be confirmed by the answers to the questions exploring the (1) existence of a forum or process to coordinate the setting of health research priorities or the (2) alignment of performance incentives to activities encouraging use of research evidence, in the survey carried out during the stakeholder meeting in Accra. The results suggest that $71.43 \%$ of respondents of the first question agree to the existence of a forum/process used to coordinate the setting up of research priorities in the health sector. On the contrary, the results of the second question suggest that approximately $60 \%$ of respondents believe that their management's participation in fora that discusses research evidence related to organisational goals is inadequate.

However, it is important to emphasise that the lack of a structured institutional mechanism within the health sector to identify research needs and communicate these to researchers does not in any way mean that policy-makers are not interested in the use of research evidence to inform policy-making. However, the findings suggest that the challenge is rather the lack of local funds to carry out research. The findings further suggest that a greater proportion of research in Ghana's health sector is funded by donors, whose objectives may not always coincide with the goals of domestic policy-makers. Thus, policy-makers are less likely to plan for their research needs given that they are less likely to receive funds domestically to carry out the research.

\section{Knowledge/research development and selection Production, synthesis and adaptation of knowledge}

In line with the conceptual framework, this section presents evidence that can be reasonably used to examine the capacity of the health sector to generate, synthesise and adapt $\mathrm{RCH}$ evidence to inform $\mathrm{RCH}$ policy. 
Information provided on the website of the GHS, in addition to discussions with key managers within the GHS, suggest that there are currently formal structures in place to ensure a coherent approach to evidence gathering and synthesis. The GHS has a Research and Development Division headed by a director. The division has three research centres (NHRC, KHRC and DHRC), in addition to three other departments responsible for (1) documentation, dissemination and advocacy, (2) ethics and research management and (3) research. As captured on the GHS website, "the key mandate of the RDD [Research and Development Division] is to generate information through relevant research to strengthen decision making, set health priorities, efficient resource allocation, and inform health interventions planning and implementation in order to deliver better health services to improve health status of the Ghanaian population" [42].

To confirm that the information above, as captured on the GHS website, is operational, a review of the websites of the three research centres was performed. The results from the review suggest extremely impressive activity in terms of completed projects, on-going projects and new projects funded by internationally reputable funding organisations such as WHO, International Labour Organisation (ILO), Bill and Melinda Gates Foundation, Rockefeller Foundation, Pfizer Corporation, Volkswagen Foundation, National Institute of Health, World Bank, West African Health Organization (WAHO), and other bilateral and multilateral donors. In addition to these projects, the three centres have impressive outputs in the form of peer-reviewed scientific publications from completed projects.

For example, the information on the website of the DHRC showed 6 completed projects, 3 on-going projects and 29 peer-reviewed scientific publications [43]. In Dodowa, 4 out of a total of 9 projects and 5 out of a total of 29 scientific publications were on $\mathrm{RCH}$. The NHRC, on the other hand, had 9 new projects, 16 on-going projects, 51 completed projects and 164 peer-reviewed scientific publications [44]. Out of a total of 73 projects in Navrongo, 17 were on $\mathrm{RCH}$, with 29 out of a total of $164 \mathrm{sci}-$ entific publications being on RCH. The KHRC had 31 completed projects and 6 on-going projects [45]. Most importantly, issues on $\mathrm{MNCH}$ featured strongly in the projects executed and scientific publications arising from the work of all the three research centres.

Besides the work of the research centres, the capacity of actors within GHS (staff and their collaborators) to produce good quality scientific evidence on $\mathrm{MNCH}$ was also examined. The results (Table 3) suggest that personnel at different levels of the GHS hierarchy are actively involved (i.e. either solely or in collaboration with others) in the production of scientific research evidence on $\mathrm{RCH}$. In addition, evidence that suggests the availability of $\mathrm{RCH}$ research, either synthesised or in raw form, that could potentially and readily be used in the formulation of $\mathrm{RCH}$ policy was also examined. Overall, 28 scientific peer-reviewed publications were examined (Table 4), including 11 on maternal health, 9 on child health and 8 on neonatal health. Out of the 28 papers, 2 were systematic reviews of the existing literature, 3 on use of evidence in policy-making and 23 on the evaluation of interventions already in operation. This is indicative of the existence of extensive evidence on $\mathrm{RCH}$ produced outside of the GHS but available to policy-makers within the GHS.

The output of the research centres is also augmented by a strong health information management system, which collects, processes and stores data from different clinical and non-clinical institutions within the GHS. Discussions with actors within and outside of the GHS indicate that the output of the health information management system constitutes strategic evidence (inputs) into different policies, including $\mathrm{RCH}$. The health information management system is equally augmented by data from several demographic surveillance systems, in addition to data from nationally representative surveys such as the GDHS and the Ghana Living Standards Survey, conducted every 5 years. Mostly, data from the three sources mentioned above are processed and presented in a format that can easily be used by policy-makers.

Clearly, the sources above point to the fact that the health sector in Ghana has enormous capacity both at the individual and organisational level to produce the much-needed evidence for policy formulation. This view is equally supported by results of responses to the questionnaire administered to participants of the Accra stakeholder meeting. The majority of respondents (as per their answers to different questions regarding the capacity of individuals within the GHS and the GHS as an institution to perform good quality research) believe that the GHS and its staff have adequate capacity to produce good quality evidence to aid policy-making.

\section{Characteristics of knowledge to be transferred}

This sub-section examines issues related to (1) the relative advantage and complexity of the knowledge to be transferred, (2) the compatibility of the knowledge to be transferred with existing beliefs and organisational norms, and (3) how the knowledge to be transferred is aligned to the needs of policy-makers.

Most of the research outputs found on the websites of the three research institutions are in the form of peer-reviewed research papers instead of alternative forms, such as policy briefs, that can be easily used by policy-makers. Although respondents to the questionnaire suggest that knowledge of policy briefs and how to use them is widespread in Ghana's health sector, the evidence 
from the study suggests that $\mathrm{RCH}$-related researchers in Ghana hardly use such means to disseminate their findings.

Although some respondents during the group discussion argued that, generally, public institutions in Ghana do not have the culture of evidence-based policy-making, this was not seen to be the case in the health sector. The difference may be due to the fact that the health sector has relatively better qualified staff who are also more experienced in research compared to other sectors in Ghana. Thus, the use of research evidence in policy-making may not be foreign to such actors. This assertion is equally confirmed by the results from the questionnaire. However, what may constitute a challenge is the extent to which the available evidence is aligned to the needs of policy-makers. As earlier on indicated, and as confirmed by the results of the questionnaire, a larger proportion of research in the health sector in general, and on $\mathrm{RCH}$ in particular, are mostly funded by donors who often have their own objectives, which may be different from those of policy-makers. Thus, besides a few circumstances where users of evidence collaborate with donors to conduct research, evidence produced from on-going research may not be aligned to the evidence/knowledge needs of policy-makers. This therefore constraints on-going efforts to improve the use of evidence to inform policy.

\section{Analysis of context}

This section deals with identified barriers and incentives to knowledge transfer at the individual, organisational and environment level. As is evident in the research output of the three research centres and the large presence of GHS staff in the RCH literature, the capacity of individuals within the health sector to produce, synthesise and potentially use research evidence in the formulation of $\mathrm{RCH}$ policies cannot be in doubt. In addition, the multiplicity of professionals (medical doctors, nurses, allied health professionals, academics, etc.) within the health sector creates internal competition with respect to knowledge production and synthesis at the individual level. It is important though to caution that the capacity to produce and use evidence at the individual level may not in itself translate into the actual use of evidence in policy formulation.

Besides the individual level capacity, the findings of the study also suggest that there are several institutional and environmental incentives that promote the use of evidence in policy formulation within Ghana's health sector. These include the sector-wide performance assessment framework linked to a set of internationally acceptable indicators that are also evidence-based, the subscription of the government through the $\mathrm{MOH}$ to major health-related international policies and conventions that are based on research evidence. This is evidenced by the several guidelines and protocols developed to ensure that actions at lower levels are consistent with the policies, protocols and conventions subscribed to. In addition, the evidence-based nature of work in the health sector makes it an environment that generally has few barriers to the use of research evidence for policy-making. This notwithstanding, major constraints to the use of evidence in policy include (1) how to ensure that research in general, and for that matter health-related research, gets the right attention at the highest level of political and administrative leadership so as to attract the right level of funding, and (2) the lack of robust and comprehensive institutional structures that ensure that the numerous health sector-related research being performed (i.e. whether specifically related to $\mathrm{RCH}$ or other relevant health issues) is aligned to the needs of policy-makers. The current disconnect between knowledge production activities and the needs of policy-makers eventually affects the extent to which policy-makers make use available knowledge to inform policy formulation. This position is confirmed by the results of the survey (questionnaire) where respondents suggest that institutional level incentives for (1) carrying out research, (2) use of research evidence implementation committees, (3) capacity to present research evidence in concise and accessible languages, and (4) capacity to synthesise different research evidence that addresses a common problem into a single document that could be attractive to policy-makers are inadequate, albeit that the required capacity and willingness to carry out good quality research exist at the individual level.

\section{Knowledge transfer activities or interventions}

This aspect of the knowledge transfer and utilisation framework looks at actual interventions put in place to facilitate/ensure the transfer of knowledge/evidence for the purposes of policy-making. In the context of Ghana's health sector, the most common intervention is post research dissemination workshops/conferences. In few instances, targeted policy briefs from completed research are disseminated to key stakeholders. However, as already indicated based on evidence from the three research centres, the use of policy briefs as a tool for ensuring the uptake of research evidence into policy in general and $\mathrm{RCH}$ in particular is rather scarce. The evidence gathered also suggests that advocacy by researchers as well as dialogue between donors and policy-makers constitute some of the channels via which research evidence on some key policy issues are discussed and consensus is reached on the possibility of adopting such evidence to aid policy formulation.

\section{Knowledge/research utilisation}

This component of the knowledge transfer process looks at the actual use of available evidence in the formulation 
of policies. Following from the evidence gathered, we examined the level and extent to which the extensive evidence available, both within and outside of the GHS, is used in the formulation of policies and guidelines. Thus, 8 policy documents ( 6 on maternal health, 1 on child health and 1 on newborn health; Table 1) were accessed and reviewed with the aim of identifying whether the contents were informed by existing scientific evidence. The findings indicated that 3 out of the 8 policy documents reviewed used an evidence-based approach (i.e. either through citation of scientific research publications, synthesis of research evidence from the academic literature or adaptation of benchmarks from key stakeholder organisations such as WHO) in developing the document. In addition to the policies, 15 standard protocols and practice guidelines (Table 2) covering maternal and adolescent health $(n=11)$, neonatal and child health $(n=1)$, general infection prevention and control $(n=1)$ and malaria prevention $(n=2)$ were also reviewed. Out of the 15 protocols and practice guidelines, 5 were evidence informed. Surprisingly, however, the policy documents and practice guidelines that suggested the use of scientific evidence did not explain the evidence generation processes and how the evidence was used in the policy/practice guidelines. Nevertheless, it is clear that the 8 policy documents and 15 protocols and practice guidelines were all developed through a consultative and participatory approach mostly involving stakeholders and professionals knowledgeable in the subject area. It is also important to state that discussions with key managers within the $\mathrm{RCH}$ division of the GHS indicated that almost all the standard protocols and practice guidelines in use are adaptations from regulators or key global institutions (WHO, UNICEF, UNFPA World Bank, etc.), which are known to rely on scientific evidence in the production of such guidelines.

In addition to the review of the policies and practice guidelines, another channel through which knowledge translation may occur (i.e. scaling up of interventions based on evidence from a pilot phase) [46, 47] was also examined. The results show (Table 5) that 10 important interventions were scaled-up based on evidence from the pilot/experiment phase. A key intervention in this direction is the Navrongo experiment (i.e. the Community Health Planning and Services (CHPS) concept). Lessons learnt from the Navrongo experiment were crucial to the scaling up of the CHPS concept, which currently constitutes a key 'Safe Motherhood' strategy in Ghana. There is also the possibility of the scaling up of capitation as a payment method across Ghana after initial piloting in the Ashanti region of the country. There is also evidence to the effect that the decision to discontinue vitamin $\mathrm{A}$ in pregnant women was based on research that found that it did not have any significant positive effect on pregnant women [48]. Again, it is on record that the Emergency Obstetric, Maternal and New Born Care study in Ghana [49] informed GHS' adoption of the maternal acceleration fund policy to facilitate $\mathrm{MNCH}$ in 2011. Additionally, evidence from the EMBRACE study is currently being used by the GHS to adopt a special maternal card to help track the progress of pregnant women so that they can adhere to their continuum of care [50]. Information from the DHRC also suggests the use of research evidence to support health policy. For example, project briefs from the DHRC show policy impact in terms of level of policy-making, type of policy, nature of policy impact, policy networks, political capital and inclusion in policy documents.

From the above, one can argue that, although a structured and robust organisational mechanism to ensure the use of scientific research evidence to inform policy formulation does not exist, actors within the health sector are relying on existing, albeit weak, institutional platforms to transfer knowledge gained from research to policy and practice.

\section{Discussion}

The information extracted in this study is herein used to answer the key question of whether Ghana's health sector has institutional structures or mechanisms in place for the production and use of evidence to inform policy formulation in general and $\mathrm{RCH}$ policies in particular.

The findings above suggest that there are currently organisational-wide structures in place for the production of $\mathrm{RCH}$ evidence. This is based on the fact that the GHS has a well-functioning and extremely active research and development division, as is evident in the number of projects and peer-reviewed scientific research publications completed. The fact that most of the research projects carried out by the research centres are funded by internationally reputable funding organisations such WHO, ILO, Bill and Melinda Gates Foundation, Rockefeller Foundation, Pfizer Corporation, Volkswagen Foundation, National Institute of Health, World Bank, WAHO, and other bilateral and multilateral donors, speaks to the quality of their research output. It is important to emphasise that quality, as used in this context, is mainly in reference to the rigorous nature of the evidence produced as opposed to coverage (i.e. relevance to policy-makers or population health needs). The rigour argument is based on the fact that funding organisations, such those indicated above, will normally ensure that research funded by them follow very rigorous standards.

Besides the output of the research centres, the findings indicate that staff from within the GHS are highly active contributors to the $\mathrm{RCH}$ literature in Ghana. Again, the existence of organisational and national level data 
repositories that can easily be accessed and used by policy-makers to aid policy formulation is ample testimony of the existence of high quality scientific research evidence that can be used to inform policy formulation. Additionally, the findings suggest that both GHS staff and other external collaborators perform scientific research within the GHS. Thus, the findings indicate to a certain systematic approach at the organisational level to enhance the production of evidence through scientific research.

The existing institutional arrangements for the production of evidence may, in some form, create opportunities for the use of evidence produced to inform policy. For example, there is evidence in the existing knowledge transfer and utilisation literature [41] that suggests that policy-makers (1) are more likely to trust evidence produced by their colleagues and therefore use such evidence to inform policy, (2) use evidence from research that involves them from planning to dissemination, (3) use evidence to inform policy if there is a window of political opportunity to do so, and (4) are more likely to use evidence to inform policy if they have a better link with the producers of evidence. Juxtaposing the results of the paper with prescriptions from the literature as indicated above, one would expect that existing GHS structures that promote the production of evidence (activities of the research centres, individuals either alone or in collaboration with others outside of the sector) by actors within the health sector may mean better credibility for such evidence and, by extension, the possibility that it will be used to inform policy. Secondly, the fact that these research centres/individuals are part of the GHS should ordinarily make it easy for those in charge of policy to have access to the producers of evidence, thereby enhancing the plausibility that available evidence will be used to inform policy formulation.

On the contrary, the results paint a mixed picture. One set of evidence indicates that systematic organisational structures as well as individual capacity that makes it relatively easy for policy-makers to use available evidence in the formulation of $\mathrm{RCH}$ policies exist. For example, project reports that capture the details (level of policy-making, type of policy, nature of policy impact, policy networks, political capital and inclusion in policy documents) of how findings of executed projects have been used in the formulation of policies is instructive. The fact that almost all the standard protocols and practice guidelines are adaptations from benchmark documents issued by regulators or key institutions such as WHO, UNICEF, UNFPA and the World Bank, which rely highly on scientific evidence in the production of such guidelines, is also important. Thus, modelling $\mathrm{RCH}$ protocols and guidelines around documents of such institutions suggests that such protocols and practice guidelines are informed by evidence.
Further, evidence on the scaling up of $\mathrm{RCH}$ interventions based on lessons learnt from the pilot phase of most of these projects is ample testimony that scientific research evidence gathered from project execution constitutes a relevant input to policy formulation and implementation. As already indicated, the scaling up of the Navrongo experiment (i.e. the CHPS concept) has had and will continue to have a profound impact on the nature and type of strategies adopted to improve $\mathrm{RCH}$ outcomes in Ghana. The possibility of scaling up capitation as a payment mechanism for Ghana's social health insurance from one region/province to national coverage is also important in this direction. Additionally, the referencing of scientific research papers in the bibliography section of some of the policies, standard protocols and practice guidelines is suggestive that findings from such papers constituted a good piece of evidence used in the formulation of the respective policies. On account of this evidence, one can suggest that capacity exists within the GHS that makes it possible to use available scientific research evidence to aid the formulation and implementation of $\mathrm{RCH}$ policies.

Nevertheless, there are findings from the study that equally suggest the existence of constraints that can compromise the ability of the GHS to use existing evidence to inform $\mathrm{RCH}$ policy. For instance, several of the policy documents and standard protocols and practice guidelines did not have any scientific research publication acknowledged in the list of references. For example, only 3 out of the 8 policy documents reviewed cited scientific research papers. In the case of the standard protocols and practice guidelines, only 5 out of the 15 documents reviewed cited the use of a scientific research paper. More importantly, those documents that cited scientific research papers did not explain the processes through which evidence emanating from these scientific papers was captured and used in either policy formulation or drafting of the standard protocols and practice guidelines. Equally important in this regard is the apparent lack of both physical and electronic evidence that shows that findings from the numerous completed projects as well as peer-reviewed publications have been converted to a form that can be easily used by policy-makers (e.g. policy briefs or policy summaries). Additionally, the divergence of donor priorities and those of policy-makers in terms of research may mean misalignment between evidence produced from research and information needed by policy-makers at a particular point in time. Thus, $\mathrm{RCH}$ evidence may be available (e.g. output of the research centres) and yet may not be useful for decision-making. It is important to caution that the policy-maker/donor divergence in research priorities may not necessarily mean a misalignment between research output of the research centres and population 
health needs, given that research undertaken by the research centres and individuals within the sector are all on issues of national relevance. Thus, the identified shortcomings should be viewed mainly as structural weaknesses within the $\mathrm{MOH}$ and its agencies, specifically the GHS, which constrain the use of evidence to inform policy formulation, and not necessarily as evidence to support the notion that policies and guidelines are not informed by existing scientific research evidence.

However, what seems to be clear from the evidence gathered so far with respect to the management of the knowledge transfer processes in Ghana's health sector and, for that matter, $\mathrm{RCH}$, is that apart from the actual knowledge production component, the rest of the process seems to be rather informal. For example, the key components of problem identification and communication, implementation of interventions and actual use of evidence do not follow any structured institutional system. Additionally, funding for research is not centrally coordinated but mostly through an arrangement between individuals or clusters of researchers within the health system and donors. It is important to emphasise that these weaknesses have collective negative implications for establishing an appropriate monitoring and evaluation framework to evaluate the knowledge production process and how it is impacting policy formulation. It is therefore not surprising that key performance indicators used to evaluate sector-wide performance in Ghana do not capture knowledge production and transfer as an activity of interest to monitor [23, 24].

\section{Strength, weaknesses, opportunities and threats}

A careful examination of the findings suggest that Ghana, compared to many sub-Saharan African countries, has made progress in producing evidence and using this to inform policy formulation. It is therefore important that the strengths of the existing system are highlighted and that appropriate measures are instituted to deal with inherent weaknesses and threats so as to take advantage of current and future opportunities to improve existing knowledge transfer processes in the health sector.

\section{Strengths and weaknesses}

The findings are suggestive that, within Ghana's health sector and particularly in the $\mathrm{RCH}$ division of the GHS, policy formulation is evidenced based. There is also enough evidence to suggest that the GHS has organisational level structures in place (the three research centres) to aid the production of the required evidence to inform policy formulation. Also important is the fact that the GHS has a critical mass of researchers who are extremely active in contributing to the production of scientific knowledge in $\mathrm{RCH}$. As earlier indicated, formal and informal structures that drive organisational learning and norms that value the importance of evidence in decision-making $[15,19]$ are key to the use of evidence in policy-making. Thus, one can argue that the systems currently in place in the GHS, though not the very best, constitute good organisational capabilities and strengths that could be leveraged upon to further improve the capacity of the GHS in general, and the $\mathrm{RCH}$ division in particular, to produce and use scientific research evidence in the formulation of $\mathrm{RCH}$ policies and guidelines. Additionally, the fact that existing norms and beliefs within the health sector are consistent with knowledge production and transfer is important for improving evidence-informed policy and practice.

The ability of researchers to summarise very complicated scientific language into simpler but easy and ready to use material by policy-makers is seen as a key facilitator of research uptake into policy [36]. In this context, one can argue that the general inability to convert most of the several scientific publications by the three research centres into policy-friendly summaries or briefs that can easily be used by policy-makers, coupled with the absence of appropriate scientific references in most of the policy documents and practice guidelines, is indicative of internal organisational weaknesses. These weaknesses refer to the absence of systematic structures that ensure that best practices are defined and followed. Given that the standard protocols and practice guidelines are adapted as indicated above, the main documents on which they are based should be adequately cited and the evidence-based processes from which the main findings were synthesised should be transparently acknowledged and referred to in the new document.

A familiar argument within the knowledge transfer and research utilisation literature has been the need to bridge the cultural and institutional gap between researchers and policy-makers, given that it constitutes a major barrier to research uptake in policy formulation [51-53]. Thus, the absence of a central structure from within the health sector to coordinate key knowledge production and transfer actors (policy-makers, researchers and donors) as well as the absence of a structured approach to define research needs and linking these to ongoing research, constitute a major weakness in the knowledge production and utilisation chain. On the basis of the above and in line with Henkel [54], we argue that policy-makers within the $\mathrm{MOH}$ and its agencies may need to work hard not only to identify problems but also to identify research that may help solve existing problems in addition to using the research output. Further, the fact that knowledge utilisation processes are not transparently defined and incorporated into organisational systems within the sector is also problematic. It is important to highlight that the absence 
of an effective monitoring and evaluation function for knowledge production and, most importantly, for transfer and utilisation constitutes a system weakness.

\section{Opportunities and threats}

Outside of the internal structures there are clear opportunities that can be exploited by the GHS to improve on its uptake of scientific research evidence as inputs to the policy formulation process. For example, the extensive network of collaborations with researchers outside of the GHS as well as access to major financiers (WHO, ILO, Bill and Melinda Gates Foundation, Rockefeller Foundation, Pfizer Corporation, Volkswagen Foundation, National Institute of Health, World Bank, WAHO and other bilateral and multilateral donors) of scientific research are all great opportunities that can be exploited to improve internal structures for producing and using good quality evidence to inform policy formulation and implementation. It is however important to emphasise that, to be able to take advantage of the opportunities enumerated, a major threat that ought to be managed well is the issue of funding. Issues around the nature and source of funding, as well as the coordination and management of such funding, are crucial. The fact that a larger proportion of funding for research in the health sector comes from outside the health sector budget is a threat to implementing any robust central coordinating mechanism for knowledge production and transfer.

It is important that as Ghana's strengths and opportunities for knowledge production and transfer are highlighted, effective and immediate steps are taken to address inherent weaknesses and threats to ensure that it improves on the current system of producing evidence and using it to inform the formulation of $\mathrm{RCH}$ policies. As clearly articulated by the participants of the Accra stakeholder meeting, improving the knowledge transfer process is a collective task that must involve all stakeholders (key among them being researchers, policy-makers and donors) with the health sector taking the lead.

\section{Conclusion}

Issues of $\mathrm{RCH}$ and $\mathrm{MNCH}$ are important areas in the strengthening health systems in developed and developing countries alike. In developing countries such as Ghana, the issue is even more critical given that health systems are generally weak and have low levels of investment and poor $\mathrm{RCH}$ indicators, especially during the 1990s. However, the implementation of pragmatic policies and commitment of health sector managers over almost two decades has 'paid off' in terms of tremendous improvement in the state of $\mathrm{RCH}$.

Nevertheless, Ghana still has challenges in $\mathrm{RCH}$ and further efforts to improve $\mathrm{RCH}$ policy-making processes are likely to yield even better results. It is therefore important that the $\mathrm{MOH}$ and its agencies, especially the GHS, take advantage of its current strengths and opportunities (strong internal knowledge production capacity, access to knowledge production networks outside of the health sector, a positive environment for the promotion of evidence-informed policy, access to major donors with the resources to fund good quality research, etc.) to confront and address the structural and organisational weaknesses inherent in the process of translating research evidence to evidenced-informed policy. Specifically, there should be deliberate efforts to mainstream research in the health sector so that an appropriate budget can be allocated to fund research. The mainstreaming of research within the sector could also help resolve the issue of the establishment of research priorities and their coordination between researchers and policy-makers.

\section{Abbreviations \\ CHPS: Community Health Planning and Services; DHRC: Dodowa Health Research Centre; EBM: Evidence-based medicine; GDHS: Ghana Demographic and Health Survey; GDP: Gross Domestic Product; HE: Health expenditure; ILO: International Labour Organisation; KHRC: Kintampo Health Research Centre; MNCH: Maternal, newborn and child health; $\mathrm{MOH}$ : Ministry of Health; NHRC: Navrongo Health Research Centre; POW: Programme of Works; $\mathrm{RCH}$ : Reproductive and child health; WAHO: West African Health Organization}

\section{Acknowledgements \\ The authors will like to acknowledge colleague faculty members who read through the manuscript and offered various suggestions to improve the paper. In the same breath, the authors acknowledge the West African Health Organization for funding the paper as well as the participants of the Accra stakeholder meeting for the comments.}

\section{Funding}

The research was funded WAHO under contract number Prog-427-DRSIS-ProgResearch-DRSIS/610/15/mbay as already indicated under the competing interests section.

\section{Availability of data and materials}

Data in the form of protocols and guidelines examined, websites and peerreviewed journal articles included are all currently publicly available and can be accessed based on the information given in the manuscript and the website of the GHS and the Ministry of Health in Ghana. Data with respect to the interviews and questionnaire are captured in the original report and briefly also in this manuscript. The original report can be accessed from the West African Health Organization on demand.

\section{Authors' contributions}

The four authors together conceptualised the paper. GAN, LV and JE developed the instruments for data collection. GAN worked with LV and JE to collect data for the paper. GAN proceeded to analyse the data collected and wrote the first draft of the paper. SI provided comments that reshaped the paper to its current form. GAN amended the paper to reflect the comments from SI and produced the current version. All authors read and approved the final manuscript.

\section{Ethics approval and consent to participate}

The study was performed under the auspices of the Ghana Health Service (GHS) and the West African Health Organization (WAHO) at the administrative level. The requisite permission to interview staff of the GHS was sought by the WAHO as part of the study. Other participants who interacted with the researchers also granted consent and were willing to participate in the interviews. Additionally, those who answered questionnaires during the stakeholder meeting in Accra also granted consent and willingly participated. Formal ethical approval was not sought given that the GHS gave administrative approval in addition to the fact that the study did not deal with any clinical staff. 


\section{Consent for publication}

Consent to publish the paper has been granted by the West African Health Organization, which is the owner of the original report. All the authors have also agreed to the publication of the paper.

\section{Competing interests}

The authors declare that the paper was funded by the West African Health Organization (WAHO), with contract number Prog-427-DRSIS-ProgResearchDRSIS/610/15/mbay. This notwithstanding, the views expressed in the paper are purely those of the authors and have not in any way been influenced by the position of WAHO. Thus, the authors together declare that they have no competing interests.

\section{Publisher's Note}

Springer Nature remains neutral with regard to jurisdictional claims in published maps and institutional affiliations.

\section{Author details}

'Department of Public Administration and Health Services Management, University of Ghana Business School, P. O. Box 72, Legon, Accra, Ghana. ${ }^{2}$ West African Health Organisation, 01 BP 153, Bobo-Dioulasso 01, Burkina Faso.

Received: 2 August 2017 Accepted: 13 July 2018 Published online: 03 August 2018

\section{References}

1. Lavis JN, Lomas J, Hamid M, Sewankambo NK. Assessing country-level efforts to link research to action. Bull World Health Organ. 2006;84:620-8.

2. Graham ID, Logan J, Harrison MB, Straus SE, Tetroe J, Caswell W, et al. Lost in knowledge translation: time for a map? J Contin Educ Heal Prof. 2006;26:13-24

3. Canadian Health Services Research Foundation. Issues in linkage and exchange between researchers and decision makers. Retrieved Novemb. 1999. https://www.cfhi-fcass.ca/migrated/pdf/event_reports/linkage_e.pdf. Accessed 20 Jun 2018.

4. Hofmeyer A, Scott C, Lagendyk L. Researcher-decision-maker partnerships in health services research: practical challenges, guiding principles. BMC Health Serv Res. 2012;12(1):280.

5. World Health Organization. World report on knowledge for better health: strengthening health systems. Geneva: WHO; 2004.

6. Ward $\mathrm{V}, \mathrm{A} H$, Hamer S. Developing a framework for transferring knowledge into action: a thematic analysis of the literature. J Health Serv Res Policy. 2009;14:156-64.

7. Lomas J, Culyer T, McCutcheon C. Conceptualizing and combining evidence for health system guidance. Ottawa: Canadian Health Services Research Foundation; 2005.

8. National Institute for Health and Clinical Excellence (NICE). Methods for the Development of NICE Public Health Guidance (2nd Edition). London: NICE; 2009.

9. Van de Ven AH, Polley DE, Garud R, Venkataraman S. The Innovation Journey. Oxford: Oxford University Press; 1999.

10. Campbell DM, Redman S, Jorm L, Cooke M, Zwi AB, Rychetnik L. Increasing the use of evidence in health policy: practice and views of policy makers and researchers. Aust New Zealand Health Policy. 2009;6:21.

11. Dobrow MJ, Goel V, Upshur REG. Evidence-based health policy: context and utilisation. Soc Sci Med. 2004;58:207-17.

12. Hanney SR, Gonzalez-Block MA, Buxton MJ, Kogan M. The utilisation of health research in policy-making: concepts, examples and methods of assessment. Health Res Policy Syst. 2003;1:2.

13. Power EJ, Tunis SR, Wagner JL. Technology assessment and public health. Annu Rev Public Health. 1994;15:561-79.

14. Oxman AD, Vandvik PO, Lavis JN, Fretheim A, Lewin S. SUPPORT Tools for evidence-informed health Policymaking (STP) 2: Improving how your organisation supports the use of research evidence to inform policymaking. Health Res Policy Syst. 2009;7:S2.

15. Hennink M, Stephenson R. Using research to inform health policy: barriers and strategies in developing countries. J Health Commun. 2005;10:163-80.

16. Roos NP, Shapiro E. From research to policy: What have we learned? Med Care. 1999;37:JS291-305.

17. Whitehead M, Petticrew M, Graham H, Macintyre SJ, Bambra C, Egan M. Evidence for public health policy on inequalities: 2: assembling the evidence jigsaw. J Epidemiol Community Health. 2004;58:817-21.
18. Stinson L, Pearson D, Lucas B. Developing a learning culture: twelve tips for individuals, teams and organizations. Med Teach. 2006;28:309-12.

19. Dowd JF. Learning organizations: an introduction. Manag Care Q. 1998;7:43-50.

20. World Bank. World Development Indicators. Washington, DC: World Bank; 2015.

21. World Health Organization. Trends in maternal mortality: 1990 to 2013 Estimates by WHO, UNICEF, UNFPA, The World Bank and the United Nations Population Division. Geneva: WHO; 2014.

22. Ghana Statistical Service (GSS) Ghana Health Service (GHS) and ICF International. The 2014 Ghana Demographic and Health Survey (DHS) Key Findings. Rockville: GSS, GHS, and ICF International; 2015.

23. Ministry of Health. Independent Annual Review of the Health Sector Programme of Works for 2007. Accra: Ministry of Health, Ghana; 2008.

24. Ministry of Health. Pulling Together, Achieving More: Independent Review of the Health Sector Programme of Work 2008. Accra: Ministry of Health, Ghana; 2009.

25. Ministry of Health. Private Health Sector Development Policy. Accra: Ministry of Health; 2012.

26. Graham ID, Tetroe J. Some theoretical underpinnings of knowledge translation. Acad Emerg Med. 2007;14:936-41.

27. Rogers EM. Diffusion of Innovations. New York: Simon and Schuster; 2010.

28. Grimshaw J, Thomas R, MacLennan G, Fraser C, Ramsay CR, Vale L, et al. Effectiveness and efficiency of guideline dissemination and implementation strategies. Health Technol Assess. 2004;8(6):iii-v. 1-72

29. Dobbins $M$, Ciliska D, Cockerill R, Barnsley J, DiCenso A. A framework for the dissemination and utilization of research for health-care policy and practice. Online J Knowl Synth Nurs. 2002;9:149-60.

30. Sevene E, Lewin S, Marino A, Woelk G, Oxman A, Matinhure S, et al. Can a drug be too cheap? The unavailability of magnesium sulphate for the treatment of eclampsia and preeclampsia in Mozambique and Zimbabwe: systems and market failures. BMJ. 2005;331(7519):765-9.

31. Trostle J, Bronfman M, Langer A. How do researchers influence decisionmakers? Case studies of Mexican policies. Health Policy Plan. 1999;14:103-14.

32. Tomson G, Paphassarang C, Jönsson K, Houamboun K, Akkhavong K, Wahlström $R$. Decision-makers and the usefulness of research evidence in policy implementation - a case study from Lao PDR. Soc Sci Med. 2005;61:1291-9.

33. Aaserud M, Lewin S, Innvaer S, Paulsen EJ, Dahlgren AT, Trommald M, et al. Translating research into policy and practice in developing countries: a case study of magnesium sulphate for pre-eclampsia. BMC Health Serv Res. 2005;5:68.

34. Albert MA, Fretheim A, Maïga D. Factors influencing the utilization of research findings by health policy-makers in a developing country: the selection of Mali's essential medicines. Health Res Policy Syst. 2007;5:2.

35. Lavis J, Davies H, Oxman A, Denis J-L, Golden-Biddle K, Ferlie E. Towards systematic reviews that inform health care management and policy-making. $J$ Health Serv Res Policy. 2005;10:35-48.

36. Innvær S, Vist G, Trommald M, Oxman A. Health policy-makers' perceptions of their use of evidence: a systematic review. J Health Serv Res Policy. 2002;7:239-44.

37. Mitton C, Adair CE, McKenzie E, Patten SB, Perry BW. Knowledge transfer and exchange: review and synthesis of the literature. Milbank Q. 2007;85:729-68.

38. Cochrane AL. Effectiveness and Efficiency: Random Reflections on Health Services. London: Nuffield Provincial Hospitals Trust London; 1972.

39. Straus SE, Graham ID, Taylor M, Lockyer J. Development of a mentorship strategy: a knowledge translation case study. J Contin Educ Heal Prof. 2008;28:117-22.

40. Lavis JN, Robertson D, Woodside JM, McLeod CB, Abelson J. How can research organizations more effectively transfer research knowledge to decision makers? Milbank Q. 2003;81:221-48.

41. Tulloch O, Mayaud P, Adu-Sarkodie Y, Opoku BK, Lithur NO, Sickle E, et al. Using research to influence sexual and reproductive health practice and implementation in Sub-Saharan Africa: a case-study analysis. Health Res Policy Syst. 2011;9:S10.

42. Ghana Health Service. Research and Development Division. Accra: Ghana health service; 2015. http://www.ghanahealthservice.org/ghs-division. php?ghs\&ghsdid=11. Accessed 20 Dec 2016.

43. Dodowa Health Research Centre. Projects Home Page. Dodowa: Dodowa health research centre; 2015. http://www.dodowa-hrc.org/new/dhrc/dhrccontent/projects/past-study. Accessed 20 Dec 2016.

44. Navrongo Health Research Centre. Past project, on-going projects and new projects. Navrongo: NHRC; 2015. http://www.navrongo-hrc.org/projs/ projects.html. Accessed 20 Dec 2016.

45. Kintampo Health Research Centre. Past, on-going and new projects. Kintampo: KHRC; 2015. http://www.kintampo-hrc.org/khrc/projects.asp. Accessed 20 Dec 2016. 
46. Uvin P, Miller D. Paths to scaling-up: alternative strategies for local nongovernmental organizations. Hum Organ. 1996;55:344-54.

47. Gaziano TA, Galea G, Reddy KS. Scaling up interventions for chronic disease prevention: the evidence. Lancet. 2007;370:1939-46.

48. Kirkwood BR, Hurt L, Amenga-Etego S, Tawiah C, Zandoh C, Danso S, et al. Effect of vitamin A supplementation in women of reproductive age on maternal survival in Ghana (ObaapaVitA): a cluster-randomised, placebocontrolled trial. Lancet. 2010;375:1640-9.

49. Ministry of Health, GHS, UNICEF, UNFPA, WHO, AMDD. National Assessment for Emergency Obstetric and Newborn Care. Accra: Ministry of Health; 2011.

50. Kikuchi K, Ansah E, Okawa S, Shibanuma A, Gyapong M, Owusu-Agyei S, et al. Ghana's Ensure Mothers and Babies Regular Access to Care (EMBRACE) program: study protocol for a cluster randomized controlled trial. Trials. 2015;16:1

51. Weiss $\mathrm{CH}$. The many meanings of research utilization. Public Adm Rev. 1979; 39:426-31.

52. Weiss CH, Bucuvalas MJ. Social Science Research and Decision-Making. Washington, DC: Columbia University Press; 1980.

53. Oliver K, Lorenc T, Innvær S. New directions in evidence-based policy research: a critical analysis of the literature. Health Res Policy Syst. 2014;12:34.

54. Kogan M, Henkel M. Government and Research: the Rothschild Experiment in a Government Department. Portsmouth: Heinemann; 1983.

55. Ghana Health Service. National Breastfeeding Policy. Accra: Ghana Health Service; 2003.

56. Ministry of Health. Ghana National Newborn Health Strategic and Action Plan 2014-2018. Accra: MoH; 2014.

57. Ministry of Health. Under 5 child Health policy: 2007-2015. Accra: MoH; 2007.

58. Ghana Health Service. Report on the Burden of Obstetric Fistula in Ghana. Accra: GHS; 2015.

59. Ministry of Health. Postpartum Hemorrhage Prevention and Management Strategy For Ghana. Accra: MoH; 2013.

60. Ministry of Health. Anti-Malaria Drug Policy for Ghana. Accra: MoH; 2007.

61. Ghana Aids Commission, USAID, UNFPA, GHS. National condom and lubricant strategy (NCLS) 2016-2020. Accra: MoH; 2015.

62. Ghana Health Service. National Family Planning Protocols. Accra: GHS; 2007.

63. Ghana Health Service, Ministry of Health. National Safe Motherhood Service Protocol. Accra: GHS; 2008.

64. Ghana Health Service, UNFPA, UKaid. Adolescent Health Info Pack. Accra: GHS; 2007.

65. Ministry of Health. What Every Pregnant Woman Should Know. Accra: MoG; 2011.

66. UNFPA. Living with Fistula. Accra: UNFPA; 2014.

67. Ministry of Health. Concise Integrated Management of Neonatal and Childhood illness. Accra: MoH; 2010.

68. Ghana Health Service. Maternal Health \& Death Audit Guidelines. Accra: GHS; 2010.

69. Ghana health service, Ghana statistical service. Ghana maternal health survey. Accra: GSS; 2007.

70. Ghana health service. Ghana health service newsletter for adolescent and young people. 3rd Edition. Accra: GHS.

71. Ministry of Health. National Policy and Guidelines for Infection Prevention and Control in Health Care Settings. Accra: $\mathrm{MoH} ; 2015$.

72. Ghana Health Service. National Reproductive Health Service Policy and Standards, 3rd Edition. Accra: GHS; 2014.

73. Ghana Health Service. Prevention and Management of Unsafe Abortion: Comprehensive Abortion Care and Services Standards \& Protocols. Accra: GHS; 2012.

74. World Health Organization. Adolescent Job Aid: A Handy Desk Reference Tool for Primary level health Workers. Geneva: WHO; 2010.

75. Ministry of Health (MOH) WHO, GLOBALFUND. Malaria in Pregnancy Training for Providers. Accra: Ministry of Health; 2010.

76. GYK G, Hill WC. A 6-year (2004-2009) review of maternal mortality at the Eastern Regional Hospital, Koforidua, Ghana. Semin Perinatol. 2012; 36(1):79-83.

77. Orish VN, Onyeabor OS, Boampong JN, Aforakwah R, Nwaefuna E, Iriemenam NC. Adolescent pregnancy and the risk of Plasmodium falciparum malaria and anaemia-A pilot study from Sekondi-Takoradi metropolis, Ghana. Acta Trop. 2012;123:244-8.

78. Tunçalp Ö, Hindin MJ, Adu-Bonsaffoh K, Adanu RM. Assessment of maternal near-miss and quality of care in a hospital-based study in Accra, Ghana. Int J Gynecol Obstet. 2013;123:58-63.
79. Abdullah F, Choo S, Hesse AAJ, Abantanga F, Sory E, Osen H, et al. Assessment of surgical and obstetrical care at 10 district hospitals in Ghana using on-site interviews. J Surg Res. 2011;171:461-6.

80. Asundep NN, Carson AP, Turpin CA, Tameru B, Agidi AT, Zhang K, et al. Determinants of access to antenatal care and birth outcomes in Kumasi, Ghana. J Epidemiol Glob Health. 2013;3:279-88.

81. Dassah ET, Adu-Sarkodie Y, Mayaud P. Estimating the uptake of maternal syphilis screening and other antenatal interventions before and after national rollout of syphilis point-of-care testing in Ghana. Int J Gynecol Obstet. 2015;130(Suppl 1):S63-9.

82. Rominski SD, Gupta M, Aborigo R, Adongo P, Engman C, Hodgson A, et al. Female autonomy and reported abortion-seeking in Ghana, West Africa. Int J Gynecol Obstet. 2014;126:217-22.

83. Hussein J, Hundley V, Bell J, Abbey M, Asare GQ, Graham W. How do women identify health professionals at birth in Ghana? Midwifery. 2005;21: 36-43.

84. Geynisman J, Latimer A, Ofosu A, Anderson FWJ. Improving maternal mortality reporting at the community level with a 4-question modified reproductive age mortality survey (RAMOS). Int J Gynecol Obstet. 2011;114: 29-32.

85. Morhe ESK, Tagbor HK, Ankobea FK, Danso KA. Reproductive experiences of teenagers in the Ejisu-Juabeng district of Ghana. Int J Gynecol Obstet. 2012; 118:137-40.

86. Okiwelu T, Hussein J, Adjei S, Arhinful D, Armar-Klemesu M. Safe motherhood in Ghana: still on the agenda? Health Policy. 2007;84:359-67.

87. Witter S, Arhinful DK, Kusi A, Zakariah-Akoto S. The experience of Ghana in implementing a user fee exemption policy to provide free delivery care. Reprod Health Matters. 2007;15:61-71.

88. Subramanian L, Cisek C, Kanlisi N, Pile JM. The Ghana vasectomy initiative: Facilitating client-provider communication on no-scalpel vasectomy. Patient Educ Couns. 2010;81:374-80.

89. Ako MA, Akweongo P. The limited effectiveness of legislation against female genital mutilation and the role of community beliefs in Upper East Region, Ghana. Reprod Health Matters. 2009;17:47-54.

90. Moyer CA, Adongo PB, Aborigo RA, Hodgson A, Engmann CM. 'They treat you like you are not a human being': maltreatment during labour and delivery in rural northern Ghana. Midwifery. 2014;30:262-8.

91. Masters SH, Burstein R, Amofah G, Abaogye P, Kumar S, Hanlon M. Travel time to maternity care and its effect on utilization in rural Ghana: a multilevel analysis. Soc Sci Med. 2013;93:147-54.

92. Baiden F, Amponsa-Achiano K, Oduro AR, Mensah TA, Baiden R, Hodgson A. Unmet need for essential obstetric services in a rural district in northern Ghana: complications of unsafe abortions remain a major cause of mortality. Public Health. 2006;120:421-6.

93. Powell-Jackson T, Hanson K, Whitty CJM, Ansah EK. Who benefits from free healthcare? Evidence from a randomized experiment in Ghana. J Dev Econ. 2014:107:305-19.

94. Sinclair D, Gyansa-Lutterodt M, Asare B, Koduah A, Andrews E, Garner P. Integrating global and national knowledge to select medicines for children: the Ghana National Drugs Programme. PLoS Med. 2013;10(5): e1001449.

95. Dassah ET, Adu-Sarkodie Y, Mayaud P. Factors associated with failure to screen for syphilis during antenatal care in Ghana: a case control study. BMC Infect Dis. 2015;15:125.

96. Cofie LE, Barrington C, Singh K, Sodzi-Tettey S, Akaligaung A. Birth location preferences of mothers and fathers in rural Ghana: Implications for pregnancy, labor and birth outcomes. BMC Pregnancy Childbirth. 2015;15:165.

97. Atuahene M, Mensah D, Adjuik M. A cross-sectional study of determinants of birth weight of neonates in the Greater Accra region of Ghana. Matern Health Neonatol Perinatol. 2015;1:1

98. Nakua EK, Sevugu JT, Dzomeku VM, Otupiri E, Lipkovich HR, OwusuDabo E. Home birth without skilled attendants despite millennium villages project intervention in Ghana: insight from a survey of women's perceptions of skilled obstetric care. BMC Pregnancy Childbirth. 2015;15:243.

99. Dalaba MA, Akweongo P, Aborigo RA, Saronga HP, Williams J, Aninanya GA, et al. Cost to households in treating maternal complications in northern Ghana: a cross sectional study. BMC Health Serv Res. 2015;15:34.

100. Manu AA, Mba CJ, Asare GQ, Odoi-Agyarko K, Asante RKO. Parent-child communication about sexual and reproductive health: evidence from the Brong Ahafo region, Ghana. Reprod Health. 2015;12:16. 
101. Achana FS, Bawah AA, Jackson EF, Welaga P, Awine T, Asuo-Mante E, et al. Spatial and socio-demographic determinants of contraceptive use in the Upper East region of Ghana. Reprod Health. 2015;12:29.

102. Adjei KK, Laar AK, Narh CT, Abdulai MA, Newton S, Owusu-Agyei S, et al. A comparative study on the availability of modern contraceptives in public and private health facilities in a peri-urban community in Ghana. Reprod Health. 2015;12:68

103. Amoakoh-Coleman M, Kayode GA, Brown-Davies C, Agyepong IA, Grobbee DE, Klipstein-Grobusch K, et al. Completeness and accuracy of data transfer of routine maternal health services data in the greater Accra region. BMC Res Notes. 2015;8:114.

104. Hall E, Sevugu J, Danso K, Adomako J, Peltzman T, Anderson FJ. A retrospective analysis of the impact of an obstetrician on delivery and care outcomes at four district hospitals in Ghana. Int J Gynaecol Obstet. 2015; 131:100-1.

105. Bawah AA, Phillips JF, Adjuik M, Vaughan-Smith M, Macleod B, Binka FN. The impact of immunization on the association between poverty and child survival: evidence from Kassena-Nankana District of northern Ghana. Scand J Public Health. 2010;38(1):95-103.

106. Adongo PB, Phillips JF, Kajihara B, Fayorsey C, Debpuur C, Binka FN. Cultural eactors constraining the introduction of family planning among the Kassena-Nankana of Northern Ghana. Soc Sci Med. 1997;45:1789-804.

107. Amoakohene MI. Violence against women in Ghana: a look at women's perceptions and review of policy and social responses. Soc Sci Med. 2004; 59:2373-85.

108. Witter S, Adjei S, Armar-Klemesu M, Graham W. Providing free maternal health care: ten lessons from an evaluation of the national delivery exemption policy in Ghana. Glob Health Action. 2009;2 https://doi.org/10. 3402/gha.v2i0.1881.

109. Oppong SA, Ntumy MY, Amoakoh-Coleman M, Ogum-Alangea D, ModeyAmoah E. Gestational diabetes mellitus among women attending prenatal care at Korle-Bu Teaching Hospital, Accra, Ghana. Int J Gynecol Obstet. 2015; 131(3):246-50.

110. Obrist M, Osei-Bonsu E, Awuah B, Watanabe-Galloway S, Merajver SD, Schmid $\mathrm{K}$, et al. Factors related to incomplete treatment of breast cancer in Kumasi, Ghana. Breast. 2014;23:821-8.

111. Shelus V, Lebetkin E, Keyes E, Mensah S, Dzasi K. Lessons from a geospatial analysis of depot medroxyprogesterone acetate sales by licensed chemical sellers in Ghana. Int J Gynecol Obstet. 2015;130(Suppl 3):E25-30.

112. Sukums F, Mensah N, Mpembeni R, Massawe S, Duysburgh E, Williams A, et al. Promising adoption of an electronic clinical decision support system for antenatal and intrapartum care in rural primary healthcare facilities in subSaharan Africa: The QUALMAT experience. Int J Med Inform. 2015;84:647-57.

113. Aborigo RA, Allotey P, Reidpath DD. The traditional healer in obstetric care: A persistent wasted opportunity in maternal health. Soc Sci Med. 2015;133:59-66.

114. de Souza D. Evidence-based approach to decision making: the inclusion of GIS as part of Ghana's health information systems. Ghana Med J. 2009:43:1.

115. Awusabo-Asare K, Abane AM, Kumi-Kyereme A. Adolescent Sexual and Reproductive Health in Ghana: A Synthesis of Research Evidence. New York: Alan Guttmacher Institute; 2004.

116. Croce-Galis M. Adolescents in Ghana: sexual and reproductive health. 2004. https://www.guttmacher.org/sites/default/files/report_pdf/rib1-04.pdf. Accessed 23 Nov 2017

117. Baker U, Tomson G, Somé M, Kouyaté B, Williams J, Mpembeni R, et al. 'How to know what you need to do': a cross-country comparison of maternal health guidelines in Burkina Faso, Ghana and Tanzania. Implement Sci. 2012:7:31.

118. Mayhew SH. Integration of STI services into FP/MCH services: health service and social contexts in rural Ghana. Reprod Health Matters. 2000:8:112-24.

119. Darteh EKM, Doku DT, Esia-Donkoh K. Reproductive health decision making among Ghanaian women. Reprod Health. 2014;11:23.

120. Sundaram A, Juarez F, Ahiadeke C, Bankole A, Blades N. The impact of Ghana's R3M programme on the provision of safe abortions and postabortion care. Health Policy Plan. 2015;30(8):1017-31.

121. Reichenbach $L$. The politics of priority setting for reproductive health: breast and cervical cancer in Ghana. Reprod Health Matters. 2002;10:47-58.

122. Amoako JF, Frempong-Ainguah F, Padmadas SS. Two decades of maternity care fee exemption policies in Ghana: have they benefited the poor? Health Policy Plan. 2016;31(1):46-55.

123. Twum-Danso NAY, Dasoberi IN, Amenga-Etego IA, Adondiwo A, Kanyoke $E_{\text {, }}$ Boadu $\mathrm{RO}$, et al. Using quality improvement methods to test and scale up a new national policy on early post-natal care in Ghana. Health Policy Plan. 2014;29:622-32

124. Friedman W, Woodman B, Chatterii M. Can mobile phone messages to drug sellers improve treatment of childhood diarrhoea? A randomized controlled trial in Ghana. Health Policy Plan. 2015;30:82-92.

125. Tawiah-Agyemang C, Kirkwood BR, Edmond K, Bazzano A, Hill Z. Early initiation of breast-feeding in Ghana: barriers and facilitators. J Perinatol. 2008;28:S46-52.

126. Edmond KM, Kirkwood BR, Amenga-Etego S, Owusu-Agyei S, Hurt LS. Effect of early infant feeding practices on infection-specific neonatal mortality: an investigation of the causal links with observational data from rural Ghana. Am J Clin Nutr. 2007:86:1126-31.

127. Manu AA, ten Asbroek A, Soremekun S, Gyan T, Weobong B, Tawiah-Agyemang $C$, et al. Evaluating the implementation of community volunteer assessment and referral of sick babies: lessons learned from the Ghana Newhints home visits cluster randomized controlled trial. Health Policy Plan. 2014;29:ii114-27.

128. Adongo PB, Kirkwood B, Kendall C. How local community knowledge about malaria affects insecticide-treated net use in northern Ghana. Trop Med Int Health. 2005;10:366-78.

129. Nyarko P, Pence B, Debpuur C. Immunization Status and Child Survival in Rural Ghana. New York: Population Council; 2001.

130. Singh K, Speizer I, Handa S, Boadu RO, Atinbire S, Barker PM, et al. Impact evaluation of a quality improvement intervention on maternal and child health outcomes in Northern Ghana: early assessment of a national scale-up project. Int J Qual Health Care. 2013;25(5):477-87.

131. Vance $G$, Janowitz B, Chen M, Boyer B, Kasonde P, Asare G, et al. Integrating family planning messages into immunization services: a cluster-randomized trial in Ghana and Zambia. Health Policy Plan. 2014;29:359-66.

132. Lei V, Friis H, Michaelsen KF. Spontaneously fermented millet product as a natural probiotic treatment for diarrhoea in young children: an intervention study in Northern Ghana. Int J Food Microbiol. 2006;110:246-53.

133. Chandramohan D, Owusu-Agyei S, Carneiro I, Awine T, Amponsa-Achiano K, Mensah $\mathrm{N}$, et al. Cluster randomised trial of intermittent preventive treatment for malaria in infants in area of high, seasonal transmission in Ghana. BMJ. 2005;331:727-33.

134. Edmond KM, Quigley MA, Zandoh C, Danso S, Hurt C, Agyei SO, et al. Diagnostic accuracy of verbal autopsies in ascertaining the causes of stillbirths and neonatal deaths in rural Ghana. Paediatr Perinat Epidemiol. 2008;22:417-29.

135. Bryce J, Friberg IK, Kraushaar D, Nsona H, Afenyadu GY, Nare N, et al. LiST as a catalyst in program planning: experiences from Burkina Faso, Ghana and Malawi. Int J Epidemiol. 2010;39:140-7.

136. Oduro AR, Wak G, Azongo D, Debpuur C, Wontuo P, Kondayire F, et al. Profile of the navrongo health and demographic surveillance system. Int J Epidemiol. 2012:41:968-76.

137. Hulton L, Matthews Z, Martin-Hilber A, Adanu R, Ferla C, Getachew A, et al. Using evidence to drive action: a "revolution in accountability" to implement quality care for better maternal and newborn health in Africa. Int J Gynecol Obstet. 2014;127:96-101.

138. Hill Z, Manu A, Tawiah-Agyemang C, Gyan T, Turner K, Weobong B, et al. How did formative research inform the development of a home-based neonatal care intervention in rural Ghana\&quest. J Perinatol. 2008;28:S38-45.

139. Moyer CA, McLaren ZM, Adanu RM, Lantz PM. Understanding the relationship between access to care and facility-based delivery through analysis of the 2008 Ghana Demographic Health Survey. Int J Gynecol Obstet. 2013;122:224-9.

140. Kapungu CT, Mensah-Homiah J, Akosah E, Asare G, Carnahan L, Frimpong MA, et al. A community-based continuum of care model for the prevention of postpartum hemorrhage in rural Ghana. Int J Gynecol Obstet. 2013;120:156-9.

141. de Savigny D, Webster J, Agyepong IA, Mwita A, Bart-Plange C, BaffoeWilmot A, et al. Introducing vouchers for malaria prevention in Ghana and Tanzania: context and adoption of innovation in health systems. Health Policy Plan. 2012;27:Iv32-43.

142. Phillips JF, Bawah AA, Binka FN. Accelerating reproductive and child health programme impact with community-based services: the Navrongo experiment in Ghana. Bull World Health Organ. 2006:84:949-55.

143. Awoonor-Williams JK, Feinglass ES, Tobey R, Vaughan-Smith MN, Nyonator FK, Jones TC. Bridging the gap between evidence-based innovation and national health-sector reform in Ghana. Stud Fam Plan. 2004;35:161-77.

144. Awoonor-Williams JK, Bawah AA, Nyonator FK, Asuru R, Oduro A, Ofosu A, et al. The Ghana Essential Health Interventions Program: a plausibility trial of 
the impact of health systems strengthening on maternal \& child survival. BMC Health Serv Res. 2013;13:S3.

145. Hill Z, Tawiah-Agyemang C, Manu A, Okyere E, Kirkwood BR. Keeping newborns warm: beliefs, practices and potential for behaviour change in rural Ghana. Trop Med Int Health. 2010;15:1118-24.

146. Awoonor-Williams JK, Sory EK, Nyonator FK, Phillips JF, Wang C, Schmitt ML. Lessons learned from scaling up a community-based health program in the Upper East Region of northern Ghana. Glob Health Sci Pract. 2013;1:117-33.

- fast, convenient online submission

- thorough peer review by experienced researchers in your field

- rapid publication on acceptance

- support for research data, including large and complex data types

- gold Open Access which fosters wider collaboration and increased citations

- maximum visibility for your research: over $100 \mathrm{M}$ website views per year

At BMC, research is always in progress.

Learn more biomedcentral.com/submissions 